\title{
Almost filling laminations and the connectivity of ending lamination space
}

\author{
DAVID GABAI
}

\begin{abstract}
We show that if $S$ is a finite type orientable surface of negative Euler characteristic which is not the 3 -holed sphere, 4 -holed sphere or 1 -holed torus, then $\mathcal{E L}(S)$ is path connected, locally path connected and cyclic.
\end{abstract}

57M50; 57R30, 51M10, 20F65

\section{Introduction}

The main result of this paper is the following:

Theorem 0.1 If $S$ is a finite type orientable surface of negative Euler characteristic which is not the 3-holed sphere, 4-holed sphere or 1-holed torus, then $\mathcal{E L}(S)$, the space of ending laminations, is path connected, locally path connected and cyclic.

$\mathcal{E} \mathcal{L}(S)$ is the space of filling, minimal geodesic laminations with the topology induced from $\mathcal{M L}(S)$ by forgetting the measure. Equivalently, it has the coarse Hausdorff topology. See Definition 1.12. Interestingly, with respect to the Hausdorff topology, $\mathcal{E} \mathcal{L}(S)$ is totally disconnected as shown by Thurston [19, Section 10] and Zhu and Bonahon [22].

Erica Klarreich [10] showed that if $S$ is hyperbolic, then the boundary of the curve complex $\mathcal{C}(S)$ is homeomorphic to the space of ending laminations on $S$. Therefore we have:

Corollary 0.2 If $S$ is a finite type orientable surface of negative Euler characteristic which is not the 3-holed sphere, 4-holed sphere or 1-holed torus, then $\partial \mathcal{C}(S)$ is path connected, locally path connected and cyclic, where $\mathcal{C}(S)$ is the curve complex of $S$.

History For the thrice-punctured sphere $S, \mathcal{C}(S)$ is trivial. It is well known that $\partial \mathcal{C}(S)$ is totally disconnected when $S$ is the 4 -holed sphere or 1 -holed torus. Peter Storm conjectured that $\partial \mathcal{C}(S)$ is path connected if $S$ is any other finite type hyperbolic surface. See Question 10 of Kent and Leininger [9]. Saul Schleimer [17] showed if 
$S$ is a once-punctured surface of genus at least two, then $\mathcal{C}(S)$ has exactly one end. With Leininger, he then showed [12] that $\mathcal{E} \mathcal{L}(S)$ is connected if $S$ is any punctured surface of genus at least two or $S$ is closed of genus at least 4. Leininger, $\mathrm{Mj}$ and Schleimer [11] showed that if $S$ is a once-punctured surface of genus at least two then $\mathcal{E L}(S)$ is both path connected and locally path connected.

The idea of the proof is as follows. Given $\mu_{0}, \mu_{1} \in \mathcal{E L}(S)$ let $\lambda_{0}$ and $\lambda_{1}$ be measured laminations whose underlying laminations are $\mu_{0}$ and $\mu_{1}$. Next, construct a path in $\mathcal{M L}(S)$ from $\lambda_{0}$ to $\lambda_{1}$. A generic PL approximation of this path will yield a new path $f_{1}:[0,1] \rightarrow \mathcal{M L}(S)$ such that for each $t, f_{1}(t)$ is an almost almost minimal almost filling measured lamination. That is, it has a sublamination $f_{1}^{*}(t)$ without proper leaves whose complement supports at most a single simple closed geodesic. A measure of the complexity of this lamination is the length of the complementary geodesic, if one exists. We now find a sequence $f_{1}, f_{2}, f_{3}, \ldots$ such that the minimal length of all complementary geodesics to the $f_{n}^{*}(t)$ 's approaches infinity as $n \rightarrow \infty$, provided such geodesics exist at all. After forgetting the measures, in the limit, we obtain the desired path in $\mathcal{E} \mathcal{L}(S)$ from $\mu_{0}$ to $\mu_{1}$. (We don't worry about whether or not the $f_{i}$ 's converge to a path in $\mathcal{M L}(S)$.) Since the final path can, in the appropriate sense, be taken arbitrarily close to the original (see Lemma 5.1), we obtain local path connectivity. Proving that the limit path is actually continuous requires control of the passage from $f_{i}$ to $f_{i+1}$. Roughly speaking, for each $t, f_{i}^{*}(t)$ lies in a $2 \epsilon_{i}$-neighborhood of the $f_{j}^{*}(t)$, if $j>i$, but not necessarily conversely, so as $j \rightarrow \infty$, the $f_{j}^{*}(t)$ 's become more and more complicated. Here distance is taken in the projective unit tangent bundle. In actuality we use discrete approximation to control the $f_{i}^{*}$ 's. For example, given $t \in[0,1]$ and $i \in \mathbb{N}$, there exists $t(i) \in[0,1]$ and a $\delta>0$ such that for $j \geq i$ and $|s-t|<\delta, f_{i}^{*}(t(i))$ lies in a $2 \epsilon_{i}$-neighborhood of $f_{j}^{*}(s)$. Here $\epsilon_{i} \rightarrow 0$ as $i \rightarrow \infty$.

As an application (of the proof of Theorem 0.1) in Section 9 we give a new construction of nonuniquely ergodic measured laminations.

Additional applications are given by Kasra Rafi and Saul Schleimer [16] who derive a number of interesting rigidity results about a surface $S$ and its curve complex $\mathcal{C}(S)$.

Denote the space of doubly degenerate Kleinian surface groups by $\operatorname{DD}(S, \partial S)$. Combining [12, Theorem 6.5] with our main result we obtain:

Corollary 9.6 $\operatorname{DD}(S, \partial S)$ is path connected, locally path connected and cyclic if $S$ is an orientable compact hyperbolic surface that is not the sphere with 3 or 4 open discs removed or the torus with an open disc removed. 
Acknowledgements We thank Saul Schleimer, Chris Leininger and Kasra Rafi for stimulating conversations and beautiful lectures on the curve complex and its boundary. Francis Bonahon, Danny Calegari, Will Cavendish, Sergio Fenley, Howie Masur, Mahan $\mathrm{Mj}$, the Referee and Peter Storm provided many useful comments and Saul Schleimer and Chris Leininger made us aware of the very interesting applications of [12] and [16]. We warmly thank all of them for their generous input.

This work was partially supported by NSF grant DMS-0504110.

\section{Basic definitions and facts}

In what follows, $S$ will denote a complete, finite volume hyperbolic surface of genus $g$ and $p$ punctures, such that $3 g+p \geq 5$.

We will assume that the reader is familiar with the elementary aspects of Thurston's theory of curves and laminations on surfaces, eg $\mathcal{L}(S)$ the space of geodesic laminations endowed with the Hausdorff topology, $\mathcal{M L}(S)$ the space of measured geodesic laminations endowed with the weak* topology and $\mathcal{P} \mathcal{M L}(S)$ projective measured lamination space, as well as the standard definitions and properties of train tracks, eg the notions of recurrent and transversely recurrent tracks, branch, fibered neighborhood, carries, etc. See Penner and Harer [15], Hamenstädt [6], Mosher [14], Canary, Epstein and Green [2] and Goldman and Thurston [4] for basic definitions and expositions of these results as well as the 1976 foundational paper by Thurston [20] and its elaboration by Fathi, Laudenbach and Poenaru [3].

All laminations in this paper will be compactly supported in $S$ and isotopic to geodesic ones. For the convenience of the reader, we recall a few of the standard definitions.

Definition 1.1 A lamination is minimal if each leaf is dense. It is filling if all complementary regions are discs or once-punctured discs. The closed complement of a lamination $\mathcal{L}$ is the metric closure of $S \backslash \mathcal{L}$ with respect to the induced path metric. A closed complementary region is a component of the closed complement. A lamination is maximal if each closed complementary region is either a 3-pronged disc or a oncepunctured monogon. $\mathcal{E} \mathcal{L}(S)$, also known as ending lamination space will denote the space of minimal filling geodesic laminations on $S$. See Definition 1.12 for a description of the topology.

If $\mathcal{L}$ is a filling geodesic lamination, then there are only finitely many ways, up to isotopy, to extend $\mathcal{L}$ to another geodesic lamination. Such extensions, including the trivial one, are called diagonal extensions. The forgetful map from $\mathcal{M L}(S)$ or $\mathcal{P M} \mathcal{L}(S)$ to $\mathcal{L}(S)$ will be denoted by $\phi$. 
Central to this paper are the following definitions.

Definition 1.2 An almost minimal almost filling lamination is a lamination $\mathcal{L}^{*}$ that is isotopic to a geodesic lamination $\mathcal{G}$ such that no leaf of $\mathcal{G}$ is proper and the closed complement supports at most a single simple closed geodesic. In other words, each closed complementary region of $\mathcal{L}^{*}$ is either a pronged disc, a once-punctured pronged disc, a twice-punctured pronged disc or a pronged annulus and there is at most one complementary region of the latter two types. Such a $\mathcal{G}$ fully supports a transverse measure. We let $\mathcal{A M L}(S) \subset \mathcal{L}(S)$ denote the set of geodesic laminations of the form $\mathcal{L}^{*} \cup \gamma$ where $\mathcal{L}^{*}$ is almost minimal almost filling and $\gamma$ is either the empty set or a simple closed geodesic. Elements of $\mathcal{A M L}(S)$ are called almost almost minimal almost filling. We abuse notation by calling such a lamination almost filling for short. If $\mathcal{L} \in \mathcal{A M L}(S)$, then $\mathcal{L}^{*}$ will denote the almost minimal almost filling sublamination. A path $f:[0,1] \rightarrow \mathcal{M L}(S)$ (or $\mathcal{P} \mathcal{M L}(S)$ ) will be called almost filling if for each $t$, $\phi(f(t)) \in \mathcal{A M L}(S)$.

Remarks 1.3 It is useful to view a geodesic lamination in three different ways. First as a foliated closed subset of $S$. Second, via its lift to $P T(S)$, the projective unit tangent bundle. Third, let $M_{\infty}$ denote $\left(S_{\infty}^{1} \times S_{\infty}^{1} \backslash \Delta\right) / \sim$, where $\Delta$ is the diagonal and $\sim$ is the equivalence relation generated by $(x, y)=(y, x)$. Since geodesics in hyperbolic 2 -space are parametrized by points in $M_{\infty}$, the preimage of a geodesic lamination in $\mathbb{H}^{2}$ can be equated with a $\pi_{1}(S)$-invariant closed subspace of $M_{\infty}$, but not necessarily conversely. We view the Hausdorff topology on $\mathcal{L}(S)$ in these three ways.

Notation 1.4 If $x \in \lambda$ and $y \in \mu$ where $\lambda, \mu \in \mathcal{L}(S)$, then $d_{P T(S)}(x, y)$ denotes distance measured in $P T(S)$. When the context is clear, the subscript will be deleted or even changed to denote distance with respect to another metric. If $\mathcal{L}$ is a geodesic lamination, then $N_{P T(S)}(\mathcal{L}, \epsilon)$ denotes the closed $\epsilon$-neighborhood of $\mathcal{L} \subset P T(S)$. The subscript or $\epsilon$ may be deleted or changed as appropriate.

If $X$ is a space, then $|X|$ denotes the number of components of $X$.

Definition 1.5 If $\tau$ is a train track, then metrize $\tau$ by decreeing that each edge has length one. A natural way to split a train track $\tau$ to $\tau_{1}$, called unzipping in the terminology of Penner and Harer [15], is as follows. Let $N(\tau)$ denote a fibered neighborhood of $\tau$ with quotient map $\pi: N(\tau) \rightarrow \tau$. Let $\sigma$ be a union of pairwise disjoint, compact, embedded curves in $N(\tau)$ transverse to the ties such that each component of $\sigma$ has at least one of its endpoints at a singular point of $N(\tau)$. See the top of Figure 1.7.4 of [15] for an example when $|\sigma|=2$. Obtain $\tau_{1}$, by deleting a 
small neighborhood of $\sigma$ from $N(\tau)$ and then contracting each resulting connected tie to a point, as in Figure 1.7.4 b of [15]. Call such an unzipping a $\sigma$-unzipping. Say the unzipping $\tau \rightarrow \tau_{1}$ has length $n$ (resp. length $\geq n$ ), if for each component $\eta$ of $\sigma$, length $(\pi(\eta)) \leq n$ with equality if exactly one endpoint lies on a singularity (resp. length $(\pi(\eta) \geq n$, unless $\eta$ has both endpoints on a singular tie.) Furthermore, each singularity of $N(\tau)$ is the endpoint of exactly one component of $\sigma$. We view the $\sigma$-unzipping to be equivalent to the $\sigma^{\prime}$-unzipping if there exists a tie preserving ambient isotopy, henceforth called a normal isotopy, of $N(\tau)$ which takes $\sigma$ to $\sigma^{\prime}$. We say that the $\sigma^{\prime}$-unzipping is an extension of the $\sigma$-unzipping, if after normal isotopy, $\sigma \subset \sigma^{\prime}$. A sequence $\tau_{1}, \tau_{2}, \ldots$ of unzippings is a full unzipping sequence if for each $N$, there exists $f(N)$ such that the induced unzipping $\tau_{1} \rightarrow \tau_{f(N)}$ is at least of length $N$. We say that $\tau$ fully carries the measured lamination $\lambda$, if $\tau$ carries $\lambda$ (ie up to isotopy $\lambda \subset N(\tau)$ and is transverse to the ties) and each tie nontrivially intersects $\lambda$.

Remarks 1.6 (i) Note that there are only finitely many length- $n$ unzippings and in particular only finitely many extensions of a length- $n$ unzipping to one of length $n+1$ and every length $\geq n$ unzipping is an extension of a length- $n$ unzipping. If $\tau_{i}$ is an unzipping of $\tau$, then $\tau$ carries $\tau_{i}$. Every splitting or shifting (as defined in [15]) is also an unzipping.

(ii) Let $\tau$ be a transversely recurrent train track in $S$. A theorem of Thurston with proof by Nat Kuhn, as in [15, Theorem 1.4.3], is that given $\epsilon>0, L>0$, there exists a hyperbolic metric on $S$ such that the geodesic curvature on $\tau$ is bounded above by $\epsilon$ and the hyperbolic length of each edge is at least $L$. Consequently, with respect to our initial hyperbolic metric, if $\tilde{\tau}$ is the preimage of $\tau$ in $\mathbb{H}^{2}$, then each bi-infinite train path $\sigma \subset \tilde{\tau}$ is a uniform quasi-geodesic in $\mathbb{H}^{2}$, hence determines an element of $M_{\infty}$. Similarly if $\tau_{i}$ is carried by $\tau$, then any bi-infinite path in $\tau_{i}$ is isotopic to one in $\tau$, hence by slightly relaxing the constant, any bi-infinite train path carried by a train track carried by $\tau$ is a uniform quasi-geodesic. Recall, that quasi-geodesics in $\mathbb{H}^{2}$ are boundedly close to geodesics.

Definition 1.7 Let $\mathcal{E}(\tau) \subset M_{\infty}$ denote those geodesics corresponding to bi-infinite train paths carried by $\tilde{\tau}$. If $\lambda$ is a geodesic lamination, then let $\mathcal{E}(\lambda) \subset M_{\infty}$ denote those geodesics which are leaves of the preimage of $\lambda$ in $\mathbb{H}^{2}$.

Remarks 1.8 By construction $\mathcal{E}(\tau)$ is $\pi_{1}(S)$-equivariant. By [15, Theorem 1.5.4] $\mathcal{E}(\tau)$ is closed in $M_{\infty}$.

Proposition 1.9 Let $\lambda_{1}, \lambda_{2}, \ldots$ be a sequence of geodesic measured laminations converging in $\mathcal{M L}(S)$ to the measured lamination $\lambda$. Let $\tau_{1}, \tau_{2}, \ldots$ be a full unzipping 
sequence such that $\tau_{1}$ is transversely recurrent and for each $i$ and for each $j \geq i, \tau_{i}$ fully carries $\lambda_{j}$. Then:

(i) The Hausdorff limit of the geodesic laminations $\phi\left(\lambda_{i}\right)$ exists and equals $\mathcal{L}$, the lamination whose preimage $\tilde{\mathcal{L}} \subset \mathbb{H}^{2}$ is comprised of the geodesics $\mathcal{E}=$ $\bigcap_{i=1}^{\infty} \mathcal{E}\left(\tau_{i}\right)$.

(ii) $\phi(\lambda)$ is a sublamination of $\mathcal{L}$.

Remarks 1.10 This is a generalization of [15, Corollary 1.7.13]. Indeed, if $\lambda=\lambda_{1}=$ $\lambda_{2}=\cdots$ then our Proposition is exactly Corollary 1.7 .13 , though stated in different language.

In our setting the limit lamination $\mathcal{L}$ need not carry a measure of full support.

Proof The proof is close to that of [15, Corollary 1.7.13]. Let $\tilde{\tau}_{i}$ (resp. $\tilde{\lambda}_{i}$ ) denote the preimage of $\tau_{i}$ (resp. $\lambda_{i}$ ) in $\mathbb{H}^{2}$. Since $\tau_{1}$ is transversely recurrent, any bi-infinite path in $\tilde{\tau}_{1}$ or its splittings is a uniform quasi-geodesic. For $j>i$, we view $\tau_{j} \subset N\left(\tau_{i}\right)$ embedded and transverse to the ties.

We first show that any geodesic $\gamma \in \mathcal{E}$ is a limit of a sequence of geodesics $\left\{\tilde{\gamma}_{i}\right\}$ where $\tilde{\gamma}_{i}$ is a leaf of $\phi\left(\tilde{\lambda}_{i}\right)$ for $i \in \mathbb{N}$. By definition $\gamma=\gamma\left(t_{1}\right)=\gamma\left(t_{2}\right)=\cdots$, where $t_{i}$ is a bi-infinite train path in $\tilde{\tau}_{i}$ and $\gamma\left(t_{i}\right)$ is the corresponding geodesic. By [15, Corollary 1.5.3], each $t_{i}$ is normally isotopic in $N\left(\tilde{\tau}_{1}\right)$ to $t_{1}$. It therefore suffices to show that each compact segment of $t_{1}$ is normally isotopic to a segment in $\tilde{\lambda}_{i}$ for all $i$ sufficiently large. This follows from the proof of [15, Lemma 1.7.9]. That argument shows that if $\tau_{i}$ is obtained from $\tau_{1}$ by a length $\geq n$ unzipping; $\omega$ is a length- $k$, $k \leq n / 2, \tilde{\tau}_{1}$-train path normally isotopic in $N\left(\tilde{\tau}_{1}\right)$ to a train path in $\tilde{\tau}_{i}$; and $\tilde{\tau}_{i}$ fully carries the lamination $\tilde{\lambda}_{i}$, then there exists a segment $\kappa$ lying in a leaf $\tilde{\gamma}_{i}$ of $\tilde{\lambda}_{i}$ that is normally isotopic in $N\left(\tilde{\tau}_{1}\right)$ to $\omega$. (Actually Penner and Harer's argument [15] requires only that $k \leq 2 n+1$, but using $k \leq n / 2$ has a somewhat easier proof.) In our setting if $n \in \mathbb{N}$, then for $i$ sufficiently large, $\tau_{i}$ is obtained from $\tau_{1}$ by a length $\geq n$ unzipping and $\tau_{i}$ carries $\lambda_{i}$. It follows that each element of $\mathcal{E}$ is the limit of a sequence $\tilde{\gamma}_{1}, \tilde{\gamma}_{2}, \ldots$ as desired. Since each $\lambda_{i}$ is a lamination, this implies that each element of $\mathcal{E}$ projects to an embedded geodesic in $S$ and the projection of no two elements cross transversely. Since each $\mathcal{E}\left(\tau_{i}\right)$ is closed, $\mathcal{E}$ is closed. Therefore the $\pi_{1}(S)$-equivariant $\mathcal{E}$ projects to a lamination in $S$, which we denote by $\mathcal{L}$.

Next we show that any convergent sequence of geodesics $\left\{\tilde{\gamma}_{i}\right\} \subset \mathbb{H}^{2}$, with $\tilde{\gamma}_{i}$ a leaf of $\tilde{\lambda}_{i}$, has limit lying in $\mathcal{E}$. Suppose $\beta_{1}, \beta_{2}, \ldots$ is a sequence of leaves respectively of $\phi\left(\tilde{\lambda}_{1}\right), \phi\left(\tilde{\lambda}_{2}\right), \ldots$ converging to the geodesic $\beta \subset \mathbb{H}^{2}$. We show that $\beta \in \mathcal{E}$. Since $\tau_{i}$ 
carries $\lambda_{j}, j \geq i$ it follows that for $j \geq i, \beta_{j} \subset \mathcal{E}\left(\tau_{i}\right)$. Since each $\mathcal{E}\left(\tau_{i}\right)$ is closed, $\beta \in \mathcal{E}\left(\tau_{i}\right)$ all $i$ and hence $\beta \in \mathcal{E}$.

We show that $\mathcal{L}$ is the Hausdorff limit of the $\phi\left(\lambda_{i}\right)$ 's. Let $U \subset P T(S)$ be a neighborhood of $\mathcal{L}$. If for some subsequence, $\phi\left(\lambda_{n_{i}}\right) \nsubseteq \mathbb{U}$, then there exists a sequence of geodesics $\beta_{n_{1}}, \beta_{n_{2}}, \ldots$ converging to $\beta \notin \mathcal{E}$ such that $\beta_{n_{i}}$ is a leaf of $\phi\left(\widetilde{\lambda}_{n_{i}}\right)$. The previous paragraph shows that $\bigcup_{i=j}^{\infty} \beta_{n_{i}} \subset \mathcal{E}\left(\tau_{k}\right)$ if $k \leq n_{j}$, which implies that $\beta \in \mathcal{E}$, a contradiction. On the other hand since each leaf of $\mathcal{L}$ is a limit of leaves of $\phi\left(\lambda_{i}\right)$ and $\mathcal{L}$ consists of finitely many minimal components plus finitely many leaves, it follows that given $\epsilon>0$, for $i$ sufficiently large $\mathcal{L} \subset N_{P T(S)}\left(\phi\left(\lambda_{i}\right), \epsilon\right)$.

To prove (ii), note that $\lambda$ is carried by each $\tau_{i}$ and hence $\mathcal{E}(\phi(\lambda)) \subset \mathcal{E}\left(\tau_{i}\right)$ all $i$ and hence $\mathcal{E}(\phi(\lambda)) \subset \mathcal{E}$.

The proof of the Proposition yields the following result.

Corollary 1.11 Under the hypothesis of Proposition 1.9, given $\epsilon>0$ there exists $N(\epsilon) \in \mathbb{N}$ so that if $i \geq N(\epsilon)$, then $\widehat{\mathcal{E}}\left(\tau_{i}\right) \subset N_{P T(S)}(\mathcal{L}, \epsilon)$, where $\widehat{\mathcal{E}}\left(\tau_{i}\right)$ denotes the union of geodesics in $S$ corresponding to elements of $\mathcal{E}\left(\tau_{i}\right)$.

Proof If not there exists $\epsilon>0$ and a sequence of geodesics $\beta_{n_{1}}, \beta_{n_{2}}, \ldots$ converging to the geodesic $\beta$ such that $\beta \notin N_{P T(\widetilde{S})}(\tilde{\mathcal{L}}, \epsilon)$ and for all $i, \beta_{n_{i}} \in \mathcal{E}\left(\tau_{n_{i}}\right)$. As in the proof the Proposition, $\beta \in \mathcal{E}\left(\tau_{i}\right)$ all $i$ and hence $\beta \in \tilde{\mathcal{L}}$, a contradiction.

The curve complex $\mathcal{C}(S)$ was introduced by Bill Harvey in 1978 [7] and it and its generalizations have subsequently played a profound role in surface topology, 3-manifold topology and geometry, algebraic topology, hyperbolic geometry and geometric group theory. In particular Howie Masur and Yair Minsky [13] showed that $\mathcal{C}(S)$ is Gromov hyperbolic. See also Bowditch [1].

Erica Klarreich showed that the Gromov boundary $\partial \mathcal{C}(S)$ of $\mathcal{C}(S)$ can be equated with $\mathcal{E L}(S)$ the space of ending laminations of $S$. These are the geodesic laminations on $S$ that are both filling and minimal, with the topology induced from $\mathcal{M L}(S)$ by forgetting the measure. More precisely, $\mathcal{E} \mathcal{L}(S)$ is given the quotient topology induced by the map $\phi: \phi^{-1}(\mathcal{E} \mathcal{L}(S)) \rightarrow \mathcal{E} \mathcal{L}(S)$ where $\phi^{-1}(\mathcal{E} \mathcal{L}(S))$ is given the subspace topology induced from $\mathcal{M L}(S)$. Ursula Hamenstädt [6] gave a direct combinatorial proof of this theorem. In the process, she introduced the coarse Hausdorff topology on $\mathcal{E} \mathcal{L}(S)$ (defined below) and showed (in the last few paragraphs of Section 1 of [6]) that this topology is equivalent to the quotient/subspace topology defined above. 
Definition 1.12 (Hamenstädt [6]) The coarse Hausdorff topology on $\mathcal{E} \mathcal{L}(S)$ is the topology such that a sequence $\mathcal{L}_{1}, \mathcal{L}_{2}, \ldots$ limits to $\mathcal{L}$ if and only if any convergent subsequence in the Hausdorff topology limits to a diagonal extension of $\mathcal{L}$. Said another way, the topology is generated by the following open sets. If $\epsilon>0$ and $\mathcal{L} \in \mathcal{E} \mathcal{L}(S)$, then an $\epsilon$-neighborhood about $\mathcal{L}$ in $\mathcal{E} \mathcal{L}(S)$ consists of all $\mu \in \mathcal{E} \mathcal{L}(S)$ such that $d_{H}\left(\mu, \mathcal{L}^{\prime}\right)<\epsilon$, for some diagonal extension $\mathcal{L}^{\prime}$ of $\mathcal{L}$.

More generally, we say that a sequence $\mathcal{L}_{1}, \mathcal{L}_{2}, \cdots \in \mathcal{L}(S)$ converges to the lamination $\mathcal{L} \in \mathcal{E} \mathcal{L}(S)$ with respect to the coarse Hausdorff topology if any convergent subsequence in the Hausdorff topology limits to a diagonal extension of $\mathcal{L}$.

The following are three characterizations of continuous paths in $\mathcal{E} \mathcal{L}(S)$.

Lemma 1.13 A function $f:[0,1] \rightarrow \mathcal{E L}(S)$ is continuous if and only if for each $t \in[0,1]$ and each sequence $\left\{t_{i}\right\}$ converging to $t, f(t)$ is the coarse Hausdorff limit of the sequence $f\left(t_{1}\right), f\left(t_{2}\right), \ldots$.

Lemma 1.14 A function $f:[0,1] \rightarrow \mathcal{E} \mathcal{L}(S)$ is continuous if and only if for each $\epsilon>0$ and $s \in[0,1]$ there exists a $\delta>0$ such that $|s-t|<\delta$ implies that the maximal angle of intersection between leaves of $f(t)$ and leaves of $f(s)$ is $<\epsilon$.

Lemma 1.15 A function $f:[0,1] \rightarrow \mathcal{E L}(S)$ is continuous if and only if for each $\epsilon>0$ and $s \in[0,1]$ there exists a $\delta>0$ such that $|s-t|<\delta$ implies that $f(t) \cap$ $N_{P T(S)}(f(s), \epsilon) \neq \varnothing$.

Remark 1.16 Let $\mathrm{S}$ be a complete hyperbolic surface that is homeomorphic to a closed surface with punctures. Let $T$ denote the corresponding compact surface with geodesic boundary, ie $T \backslash \partial T$ is homeomorphic to $S$. Then it is well known that there is a natural homeomorphism between $\mathcal{E} \mathcal{L}(S)$ and $\mathcal{E L}(S)(T)$. Similarly the homeomorphism type of $\mathcal{E} \mathcal{L}(S)$ does not depend on the hyperbolic structure.

Therefore, the main result of this paper is purely topological and is applicable to compact surfaces which are not the sphere with 3 or 4 open discs removed or the torus with an open disc removed.

\section{Combinatorics of $\alpha$-balls and almost filling PL paths}

Definition 2.1 If $\alpha$ is a simple closed geodesic in $S$, then define

$$
\widehat{B}_{\alpha}=\{\lambda \in \mathcal{M L}(S) \mid i(\alpha, \lambda)=0\},
$$

where $i(\cdot, \cdot)$ denotes intersection number and let $B_{\alpha}$ denote the projection of $\widehat{B}_{\alpha} \backslash 0$ to $\mathcal{P} \mathcal{M L}(S)$. Let $\widehat{B}_{\alpha, \beta}$ (resp. $B_{\alpha, \beta}$ ) denote $\widehat{B}_{\alpha} \cap \widehat{B}_{\beta}$ (resp. $B_{\alpha} \cap B_{\beta}$ ). 
The main result of this section, Proposition 2.2, describes the combinatorial structure of these sets. It and Corollary 2.4 are more or less well known to experts.

Proposition 2.2 If $\alpha$ is a simple closed geodesic in $S$, then $B_{\alpha}$ (resp. $\left.\widehat{B}_{\alpha}\right)$ is a compact (resp. half open) PL-ball of codimension-1 in $\mathcal{P M L}(S)$ (resp. $\mathcal{M L}(S)$ ). If $\alpha$ and $\beta$ are distinct simple closed geodesics, then $B_{\alpha, \beta}$ (resp. $\hat{B}_{\alpha, \beta}$ ) is a compact (resp. half open) PL-ball of codimension at least two in $\mathcal{P M L}(S)(\operatorname{resp} . \mathcal{M L}(S))$.

Remarks 2.3 (i) Note that $\mathcal{A M L}(S)=\phi\left(\mathcal{P M} \mathcal{L}(S) \backslash \bigcup_{\alpha \neq \beta \in \mathcal{S}} B_{\alpha, \beta}\right)$, where $\mathcal{S}$ is the set of simple closed geodesics in $S$.

(ii) By Thurston, $\mathcal{M L}(S)$ is an open ball of dimension $6 g-6+2 p$ and has a natural piecewise integral linear structure (PIL) while $\mathcal{P} \mathcal{M L}(S)$ is sphere of dimension $6 g-7+2 p$ and has a natural piecewise integral projective structure (PIP). That is, for $\mathcal{M L}(S)$ the transition functions are piecewise linear functions with integer coefficients.

Here is a brief description of the PIL structure on $\mathcal{M L}(S)$ as presented in [15, Sections 2.6 and 3.1]. A pair of pants decomposition on $S$, consists of a collection of $3 g-3+p$ pairwise disjoint simple closed geodesics. Each closed complementary region is either the sphere with 3-open discs removed, the once-punctured annulus or the twice-punctured disc. Given these curves and a parameterization of the complementary regions, construct the associated set of standard train tracks as in [15, Section 2.6]. (From now on discussion of the parametrization of the complementary regions will be suppressed.) By elementary linear algebra, if $\tau$ is a train track, then $V(\tau)$, the space of transverse measures supported by $\tau$ is a cone on a compact polyhedron in some $\mathbb{R}^{N}$. By [15, Section 2.6] we can identify $V(\tau)$ with a closed subspace of $\mathcal{M L}(S)$. Furthermore, the various $V(\tau)$ 's arising from the standard tracks glue together to give the PIL-structure on $\mathcal{M L}(S)$. In particular the maximal standard train tracks correspond to top dimensional cells and have pairwise disjoint interiors.

This structure is natural in the following sense. A different pants decomposition will give rise to a new collection of standard tracks, with change of coordinate maps given by piecewise linear integral maps. For example, by [8], one can transform any pair of pants decomposition to another via a finite sequence of elementary moves. The transition functions associated to each of the two elementary moves, were computed in Penner's thesis and are given explicitly in the Addendum of [15].

Proof of Proposition 2.2 Since $\mathcal{M L}(S)$ has a natural PIL structure, it suffices to show that $B_{\alpha}$ and $\widehat{B}_{\alpha}$ are polyhedral balls with respect to some pants decomposition. Let $\mathcal{P}$ be a pants decomposition with curves $\left(\alpha_{1}, \alpha_{2}, \ldots, \alpha_{3 g-3+p}\right)$, where $\alpha_{1}=\alpha$. Let $\lambda$ be a geodesic measured lamination. If $\alpha$ is either a leaf of $\lambda$ or lies in a 
complementary region, then with respect to global coordinates, $\lambda$ corresponds to a point $\left(0, t_{1}, i_{2}, t_{2}, i_{3}, t_{3}, \ldots, i_{3 g-3+p}, t_{3 g-3+p}\right)$, where $i_{j} \geq 0$, and $t_{j} \in \mathbb{R}$, unless $i_{j}=0$ in which case $t_{j} \geq 0$. In particular $t_{1} \geq 0$. Here $i_{j}(\lambda)=i\left(\alpha_{j}, \lambda\right)$ and $t_{j}(\lambda)$ is the twisting of $\lambda$ about $\alpha_{j}$. For a detailed exposition of global coordinates see Theorem 3.1.1 on page 152 and the last paragraph of page 174 of [15]. The connection between these coordinates and the homeomorphism between $\mathcal{M L}(S)$ and $\mathbb{R}^{6 g-6+2 p}$ is given in [15, Proposition 2.6.3 and Corollary 2.8.6].

The collection of points in $\mathcal{M L}(S)$ with first and second coordinate zero is the set of measured geodesic laminations disjoint from $\alpha$. It is an open PL-ball, denoted $\hat{S}_{\alpha}$, of dimension $6 g-6+2 p-2$ and is the union of those $V(\tau)$ 's for which $\tau$ is a subtrack of a standard train track disjoint from $\alpha$. Each element of $\widehat{B}_{\alpha}$ is of the form $t \alpha+\mathcal{G}$, where $t \geq 0$ and $\mathcal{G}$ is a measured geodesic lamination with support disjoint from $\alpha$, thus is a half open PL-ball of codimension-1, ie it is homeomorphic to $\mathbb{R}^{6 g-6+2 p-2} \times[0, \infty)=\widehat{S}_{\alpha} \times[0, \infty)$.

The quotient of $\hat{S}_{\alpha} \backslash 0$ by projectivizing is a sphere of dimension $6 g-7+2 p-2$ denoted $S_{\alpha}$. Therefore, $B_{\alpha}$ is a cone on $S_{\alpha}$ and is thus a compact PL ball of codimension-1 in $\mathcal{P} \mathcal{M L}(S)$.

If $\beta \cap \alpha=\varnothing$, then we explicitly describe $\widehat{B}_{\alpha, \beta}$ as follows. Let $\mathcal{P}$ be a pants decomposition with $\alpha_{1}=\alpha$ and $\alpha_{2}=\beta$. If $\lambda \in \widehat{B}_{\alpha, \beta}$, then $\lambda$ has coordinates $\left(0, t_{1}, 0, t_{2}, i_{3}, t_{3}, \ldots, i_{3 g-3+p}, t_{3 g-3+p}\right)$. As in the previous paragraph, when $t_{1}=$ $t_{2}=0$, this gives rise to an open PL-ball $\widehat{S}_{\alpha, \beta}$ of codimension-4 in $\mathcal{M L}(S)$ and since $t_{1}, t_{2} \geq 0$, we conclude that $\hat{B}_{\alpha, \beta}$ is a half open PL ball of codimension-2. Similarly the quotient $S_{\alpha, \beta}$ of $\hat{S}_{\alpha, \beta} \backslash 0$ is a sphere of codimension-4 in $\mathcal{P} \mathcal{M L}(S)$ and $B_{\alpha, \beta}$ is the join of a sphere and an interval (the projective classes supported on $\alpha_{1} \cup \alpha_{2}$ ), hence is a compact PL-ball of codimension-2.

If $\beta \cap \alpha \neq \varnothing$, then let $\mathcal{P}$ be a pants decomposition where for some $n \geq 2$, the collection $\left\{\alpha_{n}, \alpha_{n+1}, \ldots, \alpha_{3 g-3+p}\right\}$ is a maximal system of simple closed geodesics disjoint from both $\alpha$ and $\beta$. We can assume that $\alpha_{n}, \alpha_{n+1}, \ldots, \alpha_{k}$ are the curves that can be isotoped into $N_{S}(\alpha \cup \beta)$. Thus $B_{\alpha, \beta}$ is the join of a PL sphere of dimension $6 g-7+2 p-2 k$ with a ball of dimension $k-n$, hence is a compact ball of codimension $n+k-1 \geq 3$. A similar result holds for $\widehat{B}_{\alpha, \beta}$.

The proof of the Proposition shows:

Corollary 2.4 If $\alpha$ is a simple geodesic, then $B_{\alpha}$ is a cone of PL-sphere. If $\lambda \in \widehat{B}_{\alpha}$, then $\lambda=t \alpha+\mathcal{G}$, where $\mathcal{G}$ is a measured lamination with support disjoint from $\alpha$ and $t \geq 0$. Equality holds if and only if $\lambda \in \partial \widehat{B}_{\alpha}$.

Remark 2.5 A similar description exists for elements of $B_{\alpha, \beta}$ and $\widehat{B}_{\alpha, \beta}$. 
Definition 2.6 If $x \in \operatorname{int}\left(\widehat{B}_{\alpha}\right)$ with $\phi(x) \in \mathcal{A M L}(S)$, then define the escape ray $r_{x}$ to be the path $r:[0,1] \rightarrow \widehat{B}_{\alpha}$ by $r(t)=\left(\mathcal{L}^{*}, m_{\mathcal{L}^{*}}\right)+\left(\alpha,(1-t) m_{\alpha}\right)$. Here $\phi(x)$ is the disjoint union of the almost minimal almost filling lamination $\mathcal{L}^{*}$ and the simple closed curve $\alpha$ and $m_{\mathcal{L}^{*}}$ (resp. $m_{\alpha}$ ) denotes the transverse measure on $\mathcal{L}^{*}$ (resp. $\alpha$ ) induced from $x$. Therefore, $r(0)=x, \phi(r(1))=\mathcal{L}^{*}$ and for each $t<1, \phi(r(t))=\mathcal{L}^{*} \cup \alpha$.

Definition 2.7 A path $g:[0,1] \rightarrow \mathcal{M L}(S)$ (resp. $\mathcal{P M L}(S))$ is $P L$ almost filling if $g$ is piecewise linear, transverse to each $\widehat{B}_{\alpha}$ (resp. $B_{\alpha}$ ) and disjoint from each $\widehat{B}_{\alpha, \beta}$ (resp. $B_{\alpha, \beta}$ ) and each $\partial \widehat{B}_{\alpha}$ (resp. $\partial B_{\alpha}$ ) and hence $\phi(g(t)) \in \mathcal{A M L}(S)$ for all $t$. Also $\phi(g(t)) \in \mathcal{E} \mathcal{L}(S)$ for $t=0,1$ as well as $\phi$ of the vertices of $g$. We say that $g$ is filling (resp. almost filling) if $\phi(g(t)) \in \mathcal{E} \mathcal{L}(S)$ (resp. $\mathcal{A M L}(S))$ for all $t$.

Lemma 2.8 If $x_{0}, x_{1} \in \mathcal{M L}(S)$ with $\phi\left(x_{0}\right), \phi\left(x_{1}\right) \in \mathcal{E} \mathcal{L}(S)$, then there exists a PL almost filling path from $x_{0}$ to $x_{1}$.

Proof Since the $B_{\alpha}$ 's are countable and PL of codimension-1 and the $B_{\alpha, \beta}$ 's are countable and PL of codimension at least two, it follows that the generic PL path from $x_{0}$ to $x_{1}$ is almost filling.

Since generic PL paths are PL almost filling we have:

Lemma 2.9 Fix any metric on $\mathcal{M L}(S)$ and $\epsilon>0$. Let $h:[0,1] \rightarrow \mathcal{M L}(S)$ be continuous, such that $\phi(h(t)) \in \mathcal{E} \mathcal{L}(S)$ for $i=0,1$. Then $h$ is homotopic rel endpoints to a $P L$ almost filling path $g$ such that for each $t, d_{\mathcal{M L}(S)}(g(t), h(t))<\epsilon$.

\section{Super convergence}

The forgetful map $\phi: \mathcal{M L}(S) \rightarrow \mathcal{L}(S)$ is discontinuous, for any simple closed curve viewed in $\mathcal{M L}(S)$ is the limit of filling laminations and any Hausdorff limit of a sequence of filling laminations is filling. The content of this section is that this map is continuous in a superconvergence sense.

Definition 3.1 Let $X_{1}, X_{2}, \ldots$ be a sequence of subsets of the topological space $Y$. We say that the subsets $\left\{X_{i}\right\}$ superconverges to $X$ if for each $x \in X$, there exists $x_{i} \in X_{i}$ so that $\lim _{i \rightarrow \infty} x_{i} \rightarrow x$. In this case we say $X$ is a sublimit of $\left\{X_{i}\right\}$. 
The following is the main result of this section. Part (i) is due to Thurston [18, Proposition 8.10.3].

Proposition 3.2 (i) Suppose the measured laminations $\lambda_{1}, \lambda_{2}, \ldots$ converge to $\lambda \in \mathcal{M L}(S)$. Then $\phi\left(\lambda_{1}\right), \phi\left(\lambda_{2}\right), \ldots$ superconverges to $\phi(\lambda)$ as subsets of $P T(S)$.

(ii) If in addition $\phi(\lambda) \in \mathcal{A M L}(S)$ and $\phi\left(\lambda_{i}\right) \in \mathcal{A M L}(S)$ all $i$, then $\mathcal{L}_{1}^{*}, \mathcal{L}_{2}^{*}, \ldots$ superconverges to $\mathcal{L}^{*}$, where $\mathcal{L}_{i}^{*}$ (resp. $\mathcal{L}^{*}$ ) denotes the almost minimal almost filling sublamination of $\phi\left(\lambda_{i}\right)(\operatorname{resp} . \phi(\lambda))$.

Remark 3.3 The geodesic laminations $\mathcal{L}_{1}, \mathcal{L}_{2}, \ldots$ superconverge to $\mathcal{L}$ if and only if $\mathcal{L}$ is a sublamination of the limit of any convergent subsequence $\mathcal{L}_{n_{1}}, \mathcal{L}_{n_{2}}, \ldots$ in the Hausdorff topology.

Proof of Proposition 3.2 Part (i) follows immediately from the following Claim and Proposition 1.9.

Claim After passing to subsequence of $\left\{\lambda_{i}\right\}$ there exists a full unzipping sequence $\tau_{1}, \tau_{2}, \ldots$ of transversely recurrent train tracks such that each $\tau_{i}$ carries $\lambda$ and for $j \geq i, \tau_{i}$ fully carries $\lambda_{j}$.

Proof of Claim There are finitely many transversely recurrent train tracks such that each $x \in \mathcal{M L}(S)$ is carried by one of them, eg a system of standard train tracks. Therefore one such track $\tau_{1}$ carries infinitely many of the $\lambda_{j}$ 's. By deleting edges if necessary we can assume that $\tau_{1}$ fully carries infinitely many of these measured laminations and, after passing to subsequence, these laminations comprise our original sequence. Since each $V\left(\tau_{1}\right)$ is a closed subspace of $\mathcal{M L}(S)$ (see Remark 2.3 (ii)) and $\lambda$ is the limit of the $\lambda_{i}$ 's it follows that $\tau_{1}$ also carries $\lambda$.

Consider the finitely many train tracks obtained by a length-1 unzipping of $\tau_{1}$. Again one such track $\tau_{2}$ fully carries an infinite subset of $\left\{\lambda_{i}\right\}, i \geq 2$ and hence also $\lambda$. The claim follows by the usual diagonal argument.

We now prove part (ii). First consider the case that $\mathcal{L}^{*}$ is minimal. Let $I$ be a compact interval lying in a leaf of $\mathcal{L}^{*}$. We first show that $I$ is a sublimit of $\mathcal{L}_{i}^{*}$ 's if and only if $\mathcal{L}^{*}$ is such a sublimit. By inclusion, the latter implies the former. Conversely if $\mathcal{L}^{*}$ is not a sublimit, then there exists an $x \in \mathcal{L}^{*} \subset P T(S)$ which is not a limit point of some subsequence of the $\mathcal{L}_{i}^{*}$ 's. But the segment $I$ lies in the leaf $\sigma$ which is dense in $\mathcal{L}^{*}$. Thus, if some leaf of $\mathcal{L}_{i}^{*}$ is nearly tangent to $I$, then in $S$ it must pass very close and tangent to $\mathcal{L}^{*}$ at $x$, a contradiction. 
If $I$ is not a sublimit of $\mathcal{L}_{i}^{*}$ 's, then after passing to subsequence, there exists an open set $U \subset S$ such that $I \subset U$ and for each $i, U \cap \mathcal{L}_{i}^{*}=\varnothing$. Otherwise, the $\mathcal{L}_{i}^{*}$ 's would intersect $I$ with some definite angle, but this contradicts the fact that $I$ is a sublimit of the $\phi\left(\lambda_{i}\right)$ laminations. This argument applied to all compact intervals in leaves of $\mathcal{L}^{*}$ plus compactness of $\mathcal{L}^{*}$ implies that there exists an open set $V \subset S$ such that $\mathcal{L}^{*} \subset V$ and $V \cap \mathcal{L}_{i}^{*}=\varnothing$ all $i$ sufficiently large. This contradicts the fact that the complement of an almost minimal almost filling lamination cannot support an almost minimal almost filling lamination.

The other possibility is that $\mathcal{L}^{*}$ is the union of two minimal components $\mathcal{H}_{1}$ and $\mathcal{H}_{2}$. As above it suffices to show that if for $i=1,2, I_{i}$ is a compact interval in a leaf of $\mathcal{H}_{i}$, then $I_{1} \cup I_{2}$ is a sublimit of the $\mathcal{L}_{i}^{*}$ 's. If say $I_{1}$ was not a sublimit, then that argument also shows that there exists an open set $V \subset S$ such that $\mathcal{H}_{1} \subset V$ and for $i$ sufficiently large $V \cap \mathcal{L}_{i}^{*}=\varnothing$. Since $V$ supports infinitely many simple closed geodesics we obtain a contradiction to the fact that $\mathcal{L}_{i}^{*}$ is almost minimal almost filling.

An immediate application is the following discrete approximation lemma.

Lemma 3.4 Let $f:[0,1] \rightarrow \mathcal{M L}(S)$ be an almost filling path. Let $\epsilon>0$ and let $F \subset[0,1]$ be a finite set. Then there exists an open cover $\mathcal{I}$ of $[0,1]$ by connected sets $I(1), \ldots, I(n)$ and $t_{1}=0<t_{2}<\cdots<t_{n}=1$ such that $F \subset\left\{t_{1}, t_{2}, \ldots, t_{n}\right\}$ and $t_{i} \in I(j)$ exactly when $i=j$. Finally for all $t \in I(i)$, we have $\mathcal{L}^{*}\left(t_{i}\right) \subset N_{P T(S)}\left(\mathcal{L}^{*}(t), \epsilon\right)$. Here $\mathcal{L}^{*}(t)$ (resp. $\left.\mathcal{L}^{*}\left(t_{i}\right)\right)$ denotes the almost minimal almost filling sublamination of $f(t)$ (resp. $\left.f\left(t_{i}\right)\right)$.

\section{A criterion for constructing continuous paths in $\mathcal{E} \mathcal{L}(S)$}

Recall that our compact surface $S$ is endowed with a fixed hyperbolic metric $\rho$. Let $\left\{C_{i}\right\}_{i \in \mathbb{N}}$ denote the set of simple closed geodesics in $S$, ordered so that $i<j$ implies length $_{\rho}\left(C_{i}\right) \leq$ length $_{\rho}\left(C_{j}\right)$.

Definition 4.1 A pointed open covering $\mathcal{T}=(T, \mathcal{I})$ of the interval $[0,1]$ is a set $T=\left\{t_{1}=0<t_{2}<\cdots<t_{n}=1\right\} \subset[0,1]$ and an open covering $\mathcal{I}$ of $[0,1]$ by connected sets $I(1), I(2), \ldots, I(n)$ such that $t_{i} \in I(i)$ and if $I(j) \cap I(k) \neq \varnothing$ then $|j-k| \leq 1$. A refinement $\left(T_{2}, \mathcal{I}_{2}\right)$ of $\left(T_{1}, \mathcal{I}_{1}\right)$ has the property that $T_{1} \subset T_{2}$ and each $I_{2}(j)$ is contained in some $I_{1}(k)$. The elements of $T_{i}$ (resp. $\mathcal{I}_{i}$ ) are denoted $t_{1}^{i}, \ldots, t_{n_{i}}^{i}$, (resp. $\left.I_{i}(1), \ldots, I_{i}\left(n_{i}\right)\right)$. An infinite pointed open covering sequence $\mathcal{T}_{1}, \mathcal{T}_{2}, \ldots$ is a sequence of pointed open covers such that each term is a refinement of the preceding one. An assignment function $\mathcal{A}$ of an infinite pointed open covering sequence associates to each $s \in[0,1]$ a nested sequence of open sets $I_{1}\left(j_{1}\right) \supset I_{2}\left(j_{2}\right) \supset \cdots$ with $s \in \bigcap_{i=1}^{\infty} I_{i}\left(j_{i}\right)$ and $I_{i}\left(j_{i}\right) \in \mathcal{I}_{i}$. We let $s(i)$ denote $t_{j_{i}}^{i}$. 
Remark 4.2 It is an elementary exercise to show that any infinite pointed open covering sequence has an assignment function.

The following very technical lemma gives a criterion for constructing a continuous path in $\mathcal{E} \mathcal{L}(S)$ between two elements of $\mathcal{E} \mathcal{L}(S)$.

Lemma 4.3 Let $f_{i}:[0,1] \rightarrow \mathcal{M L}(S), i \in \mathbb{N}$ be a sequence of almost filling paths between two given points in $\mathcal{M L}(S)$ with underlying laminations in $\mathcal{E} \mathcal{L}(S)$. Let $\epsilon_{1}, \epsilon_{2}, \ldots$ be such that for all $i, \epsilon_{i} / 2>\epsilon_{i+1}>0$ and let $\mathcal{T}_{1}, \mathcal{T}_{2}, \ldots$ be an infinite pointed open cover sequence with assignment function $\mathcal{A}$. Let $\mathcal{L}_{n}^{*}(s)$ denote the almost minimal almost filling sublamination of $\phi\left(f_{n}(s)\right)$. Suppose the following conditions hold:

(sublimit) If $I_{i}(j) \cap I_{i+1}(k) \neq \varnothing$ and $t \in I_{i+1}(k)$, then $\mathcal{L}_{i}^{*}\left(t_{j}^{i}\right) \subset N_{P T(S)}\left(\mathcal{L}_{i+1}^{*}(t), \epsilon_{i}\right)$. (filling) If $s \in[0,1]$ and $m \in \mathbb{N}$, then for $n$ sufficiently large the minimal angle of intersection between leaves of $\mathcal{L}_{n}^{*}(s(n))$ with $C_{m}$ is uniformly bounded below by a constant that depends only on $m$. Also for $m$ fixed, any subsequence of $C_{m} \cap \mathcal{L}_{i}^{*}(s(i)), i \in \mathbb{N}$, has a further subsequence which converges in the Hausdorff topology on $C_{m}$ to a set with at least $4 g+p+1$ points. Here $g=\operatorname{genus}(S)$ and $p$ is the number of punctures. (Recall that $s(n)$ is given by $\mathcal{A}$.)

Then there exists a continuous path $\mathcal{L}:[0,1] \rightarrow \mathcal{E} \mathcal{L}(S)$ connecting $f_{1}(0)$ to $f_{1}(1)$ so that for $s \in[0,1], \mathcal{L}(s)$ is the coarse Hausdorff limit of $\left\{\mathcal{L}_{n}^{*}(s(n))\right\}_{n \in \mathbb{N}}$.

Proof We record the following useful and immediate fact which is a consequence of the sublimit condition and the requirement that for all $i, \epsilon_{i+1}<\epsilon_{i} / 2$.

Claim If $t \in I_{i}(j)$ and $n \geq i$, then $\mathcal{L}_{i}^{*}\left(t_{j}^{i}\right) \subset N_{P T(S)}\left(\mathcal{L}_{n}^{*}(t), 2 \epsilon_{i}\right)$.

This claim demonstrating the utility of the sublimit condition shows how a given $f_{i}$ imposes structure on $f_{k}$ for all $k \geq i$ and is the key to the proof of continuity. A similar result together with the filling condition are the main ingredients for showing that $\mathcal{L}(s)$ is the coarse Hausdorff limit of the $\mathcal{L}_{n}^{*}(s(n))$ 's. Here are the details.

Fix $s$. We construct the minimal and filling $\mathcal{L}(s)$. Given $s \in[0,1]$, let $I_{1}\left(j_{1}\right) \supset$ $I_{2}\left(j_{2}\right) \supset \cdots$ be the nested sequence of $I_{i}(j)$ intervals given by $\mathcal{A}$ which contain $s$. After passing to a subsequence we can assume that $\left\{\mathcal{L}_{n_{i}}^{*}\left(s\left(n_{i}\right)\right)\right\}$ converges in the Hausdorff topology to the lamination $\mathcal{L}^{\prime} . \mathcal{L}^{\prime}$ contains no closed leaves since the filling condition implies that $\mathcal{L}^{\prime}$ is transverse to every simple closed geodesic. Let $\mathcal{L}$ be a minimal sublamination of $\mathcal{L}^{\prime}$. If $\mathcal{L}$ is not filling, then there exists a simple closed geodesic $C$, disjoint from $\mathcal{L}$ that can be isotoped into any neighborhood of $\mathcal{L}$ 
in $S$. An elementary topological argument shows that $\left|C \cap \mathcal{L}^{\prime}\right|<4 g+p+1$. This contradicts the second part of the filling condition. We now show that $\mathcal{L}$ is independent of subsequence. Let $\mathcal{L}_{1} \in \mathcal{E} \mathcal{L}(S)$ be a lamination that is the coarse Hausdorff limit of the subsequence $\left\{\mathcal{L}_{m_{i}}^{*}\left(s\left(m_{i}\right)\right)\right\}$ and let $\epsilon>0$. If $x \in \mathcal{L}_{1}$, then there exists a sequence $x_{m_{i}} \in \mathcal{L}_{m_{i}}^{*}\left(s\left(m_{i}\right)\right)$ such that $\lim _{i \rightarrow \infty} x_{m_{i}}=x$ where the limit is taken in $\operatorname{PT}(S)$. The sublimit property implies that for all $k>m_{i}$, there exists $y_{k} \in \mathcal{L}_{k}^{*}(s(k))$ such that $d_{P T(S)}\left(x_{m_{i}}, y_{k}\right)<\epsilon_{m_{i}}+\epsilon_{m_{i}+1}+\cdots+\epsilon_{k-1}<2 \epsilon_{m_{i}}$. As $i \rightarrow \infty, \epsilon_{m_{i}} \rightarrow 0$ and $m_{i} \rightarrow \infty$ so it follows that $\mathcal{L}_{1} \subset \mathcal{L}^{\prime}$. Since $\mathcal{L}_{1}, \mathcal{L} \in \mathcal{E} \mathcal{L}(S)$, we conclude that $\mathcal{L}=\mathcal{L}_{1}$. Denote $\mathcal{L}$ by $\mathcal{L}(s)$. We have shown that $\mathcal{L}(s)$ exists and satisfies the coarse Hausdorff limit condition of the conclusion.

We apply Lemma 1.15 to show that $f$ is continuous. Fix $s \in[0,1]$ and let $I_{1}\left(j_{1}\right) \supset$ $I_{2}\left(j_{2}\right) \supset \cdots$ be the nested sequence of $I_{i}(j)$ intervals given by $\mathcal{A}$ which contain $s$. Let $U$ open in $P T(S)$ such that $\mathcal{L}(s) \subset U$. Pick $z \in \mathcal{L}(s)$ and let $\epsilon>0$ such that $N_{P T(S)}(z, \epsilon) \subset U$. We will show that for $t$ sufficiently close to $s, \mathcal{L}(t) \cap N_{P T(S)}(z, \epsilon) \neq$ $\varnothing$. Choose $i$, such that $\epsilon_{i}<\epsilon / 2$. Since $\mathcal{L}(s)$ is the coarse Hausdorff limit of the $\mathcal{L}_{k}^{*}(s(k))$ it follows that for $k$ sufficiently large there exists an $x_{k} \in \mathcal{L}_{k}^{*}(s(k))$ so that $d_{P T(S)}\left(x_{k}, z\right)<\epsilon_{i}$. Assume that $k>i$. The Claim implies that if $t \in I_{k}\left(j_{k}\right)$, then for every $n \geq k$ there exists $y_{n} \in \mathcal{L}_{n}^{*}(t)$ such that $d_{P T(S)}\left(x_{k}, y_{n}\right) \leq 2 \epsilon_{k}$ and hence for some diagonal extension $\mathcal{L}^{\prime}(t)$ of $\mathcal{L}(t)$ there exists $y \in \mathcal{L}^{\prime}(t)$ with $d_{P T(S)}\left(y, x_{k}\right) \leq 2 \epsilon_{k}<\epsilon_{i}$ and hence $d_{P T(S)}(y, z)<2 \epsilon_{i}<\epsilon$.

Assume that $\epsilon$ is sufficiently small so that the $\epsilon$-disc $D \subset S$, centered at $z$, is embedded. Since $\mathcal{L}(s)$ is minimal and has infinitely many leaves, the above argument shows that given $N \in \mathbb{N}$, there exists a $\delta>0$ so that $|t-s|<\delta$ implies for some diagonal extension $\mathcal{L}^{\prime}(t)$ of $\mathcal{L}(t)$, the intersection $\mathcal{L}^{\prime}(t) \cap D$ contains at least $N$ arcs extremely close to and nearly parallel to the local leaf of $\mathcal{L}(s)$ through $z$. Extremely close and nearly parallel mean that if $\sigma$ is a geodesic path lying between a pair of these arcs, then $\sigma \cap N_{P T(S)}(z, \epsilon) \neq \varnothing$. It is possible that all of these $N$ arcs lie in $\mathcal{L}^{\prime}(t) \backslash \mathcal{L}(t)$, however, if $N$ is sufficiently large and $\gamma \subset D$ is a geodesic transverse to these $N$ arcs, then $\gamma \cap \mathcal{L}(t) \neq \varnothing$. Indeed, since the number of diagonal leaves of $\mathcal{L}^{\prime}(t)$ is uniformly bounded (as a function of $S$ ) by say $M$, then $N=M+1$ suffices, for if $\gamma$ is disjoint from $\mathcal{L}(t)$ and hits a given leaf of $\mathcal{L}^{\prime}(t)$ twice, then $\left.\mathcal{L}^{(} t\right)$ is not filling, a contradiction. Therefore, $\mathcal{L}(t) \cap N_{P T(S)}(z, \epsilon) \neq \varnothing$.

\section{$5 \mathcal{E L}(S)$ is path connected}

Let $\mu_{0}, \mu_{1} \in \mathcal{E L}(S)$ and $\lambda_{0}, \lambda_{1} \in \mathcal{M L}(S)$ such that $\phi\left(\lambda_{i}\right)=\mu_{i}, i=0,1$. In this section we produce a sequence of PL almost filling paths in $\mathcal{M L}(S)$ that satisfy Lemma 4.3, from which we obtain a path from $\mu_{0}$ to $\mu_{1}$. 
The idea is as follows. By Lemma 2.8 there exists a PL almost filling path $f_{1}:[0,1] \rightarrow$ $\mathcal{M L}(S)$ from $\lambda_{0}$ to $\lambda_{1}$. If that path is disjoint from each $\widehat{B}_{C_{i}}$, then $\phi \circ f_{1}$ is the desired path. If not, it intersects the various $\widehat{B}_{C_{i}}$ 's transversely. Since each $B_{C_{i}}$ is a compact PL-ball of codimension-1 in a sphere of dimension at least 3, it follows that $f_{1}$ can be homotoped off of any $\widehat{B}_{C_{i}}$. Our sequence of functions is obtained by iteratively homotoping $f_{1}$ off of $\widehat{B}_{C_{1}}, \widehat{B}_{C_{2}}, \ldots$ The crucial observation is that if the support of the homotopy of $f_{i}$ off of $\widehat{B}_{C_{i}}$ is done very close to the escape ray (See Definition 2.6.) and $f_{i+1}$ is the resulting function, then Proposition 3.2 (ii) implies that $\mathcal{L}_{i+1}^{*}(t) \subset N_{P T(S)}\left(\mathcal{L}_{i}^{*}(t), \epsilon\right)$, where $\mathcal{L}_{n}^{*}(s)$ denotes the almost minimal almost filling sublamination of $\phi\left(f_{n}(s)\right)$. In other words $\mathcal{L}_{i+1}^{*}(t)$ roughly looks like $\mathcal{L}_{i}^{*}(t)$ plus extra stuff. Hence for each $m<n, C_{m} \cap \mathcal{L}_{n-1}^{*}(t) \neq \varnothing$ implies that $C_{m} \cap \mathcal{L}_{n}^{*}(t) \neq \varnothing$. Thus, if $f_{n-1}$ has been constructed to avoid $\widehat{B}_{C_{1}}, \widehat{B}_{C_{2}}, \ldots, \widehat{B}_{C_{n-1}}$ the newly constructed $f_{n}$ continues to avoid these balls. The technical work entails controlling the homotopies so that the sublimit and filling conditions hold.

Let $f_{1}:[0,1] \rightarrow \mathcal{M L}(S)$ from $\lambda_{0}$ to $\lambda_{1}$ be a PL almost filling path. Fix $\epsilon_{1}>0$. Let $\mathcal{L}_{1}^{*}(t)$ denote the almost minimal almost filling sublamination of $\phi\left(f_{1}(t)\right)$. By Lemma 3.4, there exists a pointed open cover $\widehat{\mathcal{T}}_{1}=\left(T_{1}, \widehat{\mathcal{I}}_{1}\right)$ with $T_{1}=\left\{t_{1}^{1}, \ldots, t_{n}^{1}\right\}$ and $\widehat{\mathcal{I}}_{1}=\left\{\widehat{I}_{1}(1), \ldots, \widehat{I}_{1}(n)\right\}$ such that for $t \in \widehat{I}_{1}(j), \mathcal{L}_{1}^{*}\left(t_{j}^{1}\right) \subset N\left(\mathcal{L}_{1}^{*}(t), \epsilon_{1} / 2\right)$. By shrinking the $\hat{I}_{1}(j)$ 's we can assume that for each $k$ some open neighborhood $Y(k)$ of $t_{k}^{1}$ nontrivially intersects only $\widehat{I}_{1}(k)$. By further shrinking the $\widehat{I}_{1}(k)$ 's we get a new open cover $\mathcal{I}_{1}=\left\{I_{1}(1), \ldots, I_{1}(n)\right\}$ and $\eta_{1}>0$ such that for all $j, N_{[0,1]}\left(I_{1}(j), \eta_{1}\right) \subset$ $\widehat{I}_{1}(j)$. We let $\mathcal{T}_{1}=\left(T_{1}, \mathcal{I}_{1}\right)$. Since $f_{1}$ is transverse to the $\widehat{B}_{\alpha}$ 's, it intersects $\widehat{B}_{C_{1}}$ in at most a finite set of points. By including these points in $F$ (notation as in Lemma 3.4) we can assume that these points were included in $T_{1}$. Without loss we will assume that there exists a unique point $x$ of intersection, and $f_{1}\left(t_{p}^{1}\right)=x$.

We homotope $f_{1}$ to $f_{2}$ so that $f_{2} \cap \widehat{B}_{C_{1}}=\varnothing$ as follows. First homotope $f_{1}$ to $f_{1.1}$, via a homotopy supported in $Y(p)$ so that the image of $f_{1.1}$ is the image of $f_{1}$ together with the escape ray $r_{x}$. That is, the path $f_{1.1}$ follows along the image of $f_{1}$ until it hits $x$, then goes all the way out along $r_{x}$ and then back along $r_{x}$ to $x$ and then continues away from $\hat{B}_{C_{1}}$ as does $f_{1}$. Since $\hat{B}_{C_{1}}$ is PL ball of codimension-1 a very small perturbation of $f_{1.1}$ yields $f_{1.2}$ disjoint from $\widehat{B}_{C_{1}}$. A generic PL approximation of $f_{1.2}$ yields a PL almost filling path $f_{2}$ disjoint from $\hat{B}_{C_{1}}$.

Since $\mathcal{L}_{1}^{*}\left(t_{p}^{1}\right)$ is the almost minimal almost filling lamination associated to any point on $r_{x}$, Proposition 3.2 plus compactness implies that if the nontrivial tracks of the homotopy from $f_{1}$ to $f_{2}$ lay sufficiently close to $r_{x}$, then for $s \in Y(p), \mathcal{L}_{1}^{*}\left(t_{p}^{1}\right) \subset$ $N\left(\mathcal{L}_{2}^{*}(s), \epsilon_{1}\right)$. Since $f_{2}([0,1]) \cap \widehat{B}_{C_{1}}=\varnothing, C_{1}$ nontrivially intersects each $\mathcal{L}_{2}^{*}(t)$ transversely. Since the homotopy from $f_{1}$ to $f_{2}$ is supported in $Y(p), f_{2}$ satisfies the 
sublimit property (ie the sublimit property holds for $i+1=2$ ) provided that the, to be defined, cover $\mathcal{I}_{2}$ has the property that if $I_{2}(j) \cap I_{1}(k) \neq \varnothing$, then $I_{2}(j) \subset \widehat{I}_{1}(k)$.

The next three paragraphs explain how to choose $\epsilon_{2}$ so the (to be constructed) $f_{i}$ 's will satisfy the filling property with respect to $C_{1}$, assuming these $f_{i}$ 's satisfy the sublimit property. By compactness of $[0,1]$ and Proposition 3.2 there exists a $\psi>0$ that is a uniform lower bound, independent of $t$, for the maximal angle of intersection between a $\mathcal{L}_{2}^{*}(t)$ and $C_{1}$. Furthermore, there exists $\kappa>0$ so that if $\mathcal{L}$ is a geodesic lamination, $t \in[0,1]$ and $\mathcal{L}_{2}^{*}(t) \subset N(\mathcal{L}, \kappa)$, then $\mathcal{L} \cap C_{1} \neq \varnothing$ and the maximal angle of intersection is at least $\psi / 2$. Since $C_{1}$ has bounded length, the lower bound $\psi / 2$ on maximal angle of intersection of a geodesic lamination with $C_{1}$ implies a positive lower bound $\phi$ on minimal angle of intersection.

Let $N=4 g+p+1$, where $g=\operatorname{genus}(S)$ and $p$ is the number of punctures. Since for every $t,\left|\mathcal{L}_{2}^{*}(t) \cap C_{1}\right|=\infty$, the angle condition, compactness and Proposition 3.2 imply that there exists a $\psi^{\prime}>0$ and $\kappa^{\prime}<\kappa$ so that each $\mathcal{L}_{2}^{*}(t) \cap \mathcal{C}_{1}$ contains $N$ points, any two of which are at least distance $\psi^{\prime}$ apart, measured along $C_{1}$. Furthermore, if $\mathcal{L}$ is a geodesic lamination with $\mathcal{L}_{2}^{*}(t) \subset N\left(\mathcal{L}, \kappa^{\prime}\right)$, then $\mathcal{L} \cap C_{1}$ contains $N$ points, any two of which are $\psi^{\prime} / 2$ apart on $C_{1}$. Pick $\epsilon_{2}<\min \left(\kappa^{\prime} / 2, \epsilon_{1} / 2\right)$.

If the, to be defined, $f_{i}$ 's and $I_{i}(j)$ 's satisfy the sublimit property, $\epsilon_{2}$ is as above and the $\epsilon_{i}$ 's are chosen so that $\epsilon_{i+1}<\epsilon_{i} / 2$, then the $f_{i}$ 's will satisfy the filling property with respect to $C_{1}$, independent of choice of assignment function. To see this, first pick $s \in[0,1]$ and suppose that $s \in I_{2}(q)$. If $m \geq 2$ and $s \in I_{m}(j) \in \mathcal{I}_{m}$, then by the sublimit property, $\mathcal{L}_{2}^{*}\left(t_{q}^{2}\right) \subset N_{P T(S)}\left(\mathcal{L}_{m}^{*}\left(t_{j}^{m}\right), \epsilon_{m-1}+\cdots+\epsilon_{3}+\epsilon_{2}\right) \subset N_{P T(S)}\left(\mathcal{L}_{m}^{*}\left(t_{j}^{m}\right), 2 \epsilon_{2}\right) \subset$ $N_{P T(S)}\left(\mathcal{L}_{m}^{*}\left(t_{j}^{m}\right), \kappa^{\prime}\right)$ and hence $\mathcal{L}_{m}^{*}\left(t_{j}^{m}\right) \cap C_{1}$ contains $N$ points spaced in $C_{1}$ at least $\psi^{\prime} / 2$ apart and the minimal angle of intersection of $\mathcal{L}_{m}^{*}\left(t_{j}^{m}\right)$ with $C_{1}$ is bounded below by $\phi$. The filling property for $C_{1}$ follows.

Let $X_{2}=\left\{t \in[0,1] \mid f_{2}(t) \cap \widehat{B}_{C_{2}} \neq \varnothing\right\}$. Apply Lemma 3.4 to $f_{2}$ using $\epsilon=\epsilon_{2}$ and $F=T_{1} \cup X_{2}$ (henceforth called $\left.F_{2}\right)$ to obtain $\widehat{\mathcal{T}}_{2}=\left(T_{2}, \widehat{\mathcal{I}}_{2}\right)$. Again we can assume that for each $k$, some open neighborhood of $t_{k}^{2}$ nontrivially intersects only $\widehat{I}_{2}(k)$. By adding appropriate extra points to $F_{2}, \widehat{I}_{2}(j) \cap I_{1}(k) \neq \varnothing$ implies $\widehat{I}_{2}(j) \subset \widehat{I}_{1}(k)$. Finally shrink $\hat{\mathcal{I}}_{2}$ as before to obtain $\mathcal{I}_{2}$ and $\mathcal{T}_{2}$.

Use $\epsilon_{2}$ and $C_{2}$ to construct $f_{3}$ from $f_{2}$ in the same way that $f_{2}$ was constructed from $f_{1}$. Again the resulting $f_{3}$ satisfies the sublimit condition provided $I_{3}(j) \cap I_{2}(k) \neq \varnothing$ implies $I_{3}(j) \subset \widehat{I}_{2}(k)$. Choose $\epsilon_{3}$ as above so that subsequently constructed $f_{i}$ 's will satisfy the filling condition with respect to $C_{2}$. Define and construct $\widehat{\mathcal{T}}_{3}$ and $\mathcal{T}_{3}$ analogous to the constructions of $\widehat{\mathcal{T}}_{2}$ and $\mathcal{T}_{2}$.

The proof of path connectivity is now completed by induction. 
Our proof shows that in the sublimit sense any path in $\mathcal{M L}(S)$ connecting points in $\mathcal{E} \mathcal{L}(S)$ can be perturbed rel endpoints by an arbitrarily small amount to a path in $\mathcal{E} \mathcal{L}(S)$. More precisely we have:

Lemma 5.1 If $f:[0,1] \rightarrow \mathcal{M L}(S)$ is a PL almost filling path with $\phi(f(0), \phi(f(1))$ in $\mathcal{E} \mathcal{L}(S), \epsilon>0$ and $\delta>0$, then there exists a path $g:[0,1] \rightarrow \mathcal{E} \mathcal{L}(S)$ from $\phi(f(0))$ to $\phi(f(1))$ such that for each $s \in[0,1]$ there exists $t \in[0,1]$ with $|t-s|<\delta$ such that, $\mathcal{L}^{*}(t) \subset N_{P T(S)}\left(g(s)^{\prime}, \epsilon\right)$, for some diagonal extension $g(s)^{\prime}$ of $g(s)$. Here $\mathcal{L}^{*}(t)$ denotes the almost minimal almost filling sublamination of $\phi(f(t))$.

Proof Apply the above proof with $f_{1}=f, \epsilon_{1}<\epsilon / 4$ and the mesh of $F<\delta$. Pick $s \in[0,1]$. With notation as in Lemma 4.3, if $n$ is sufficiently large $\mathcal{L}_{n}^{*}(s(n)) \subset$ $N_{P T(S)}\left(\mathcal{L}^{\prime}(s), \epsilon_{1}\right)$ for some diagonal extension $\mathcal{L}^{\prime}(s)$ of $\mathcal{L}(s)$. By the sublimit property $\mathcal{L}_{1}^{*}(s(1)) \subset N_{P T(S)}\left(\mathcal{L}_{n}^{*}(s(n)), 2 \epsilon_{1}\right)$. Since $\delta \leq \operatorname{mesh} T_{1},|s-s(1)| \leq \delta$ and the result follows.

\section{$6 \mathcal{E L}(S)$ is locally path connected}

Theorem 6.1 If $S$ is a finite type orientable surface of negative Euler characteristic which is not the 3 -holed sphere, 4 -holed sphere or 1-holed torus, then $\mathcal{E} \mathcal{L}(S)$ is locally path connected.

In this section we show that if $S$ satisfies the hypothesis of Theorem 0.1 , then $\mathcal{E} \mathcal{L}(S)$ is locally path connected. It suffices to show that if $\mu \in U$ an open set in $\mathcal{E} \mathcal{L}(S)$, then there exists an open set $V \subset U$ containing $\mu$ such that for each $\mathcal{L} \in V$ there is a path in $U$ from $\mu$ to $\mathcal{L}$. Therefore, the path component $W$ of $U$ containing $\mu$ is open and path connected.

In order to free the reader of nasty notation and slightly extra technical detail, we first consider the case that $\mu$ is maximal, ie every complementary region is either a 3 -pronged disc or a once-punctured monogon.

Let $\tau_{1}$ be a transversely recurrent train track that fully carries $\mu$ and let $\tau_{1}, \tau_{2}, \tau_{3}, \ldots$ be the unique sequence of train tracks, such that $\tau_{i+1}$ is a length $-i$ unzipping of $\tau_{1}$ and $\tau_{i+1}$ fully carries $\mu$. Let $E_{i}=\left\{\mathcal{G} \in \mathcal{E} \mathcal{L}(S) \mid \mathcal{G}\right.$ is fully carried by $\left.\tau_{i}\right\}$. It is well known that each $E_{i}$ is open in $\mathcal{E} \mathcal{L}(S)$ and $\bigcap E_{i}=\mu$. The latter also follows from Corollary 1.11 and the former is readily verified by observing that any $\mathcal{H} \in \mathcal{E} \mathcal{L}(S)$ sufficiently close in the Hausdorff topology to a diagonal extension of $\mathcal{G} \in E_{i}$ is also carried by $\tau_{i}$. By dropping the first terms of the $\tau_{i}$ sequence, we can assume that if $\mathcal{G} \in E_{1}$, then $\mathcal{G} \subset U$. 
There exists $W_{2}$ open in $P T(S)$ so that $\mu \subset W_{2}$ and if $\mathcal{L} \in \mathcal{L}(S)$ with $\mathcal{L} \cap W_{2} \neq \varnothing$, then every sublamination of $\mathcal{L}$ is fully carried by $\tau_{1}$. This follows from [13], though for completeness we outline an argument here. First observe that there exists $W_{1}$ open in $\operatorname{PT}(S)$ so that $\mu \subset W_{1}$ and if $\mathcal{L} \in \mathcal{L}(S)$ with $\mathcal{L} \cap W_{1} \neq \varnothing$, then $\mathcal{L}$ is fully carried by $\tau_{1}$. If no such $W_{2}$ exists, then there exists a sequence of laminations $\mathcal{L}_{1}, \mathcal{L}_{2}, \ldots$ with sublaminations $\mathcal{G}_{1}, \mathcal{G}_{2}, \ldots$ such that $\mathcal{L}_{i} \cap N_{P T(S)}(\mu, 1 / i) \neq \varnothing$, but $\mathcal{G}_{i} \cap W_{1}=\varnothing$. After passing to subsequence we can assume the $\mathcal{G}_{i}$ 's converge in the Hausdorff topology to $\mathcal{G}$ where $\mathcal{G} \cap W_{1}=\varnothing$. Since $\mu$ is filling, this implies that $\mathcal{G} \cap \mu \neq \varnothing$ and $\mathcal{G}$ is transverse to $\mu$. On the other hand $\mu$ is approximated arbitrarily well by segments lying in leaves of $\mathcal{L}_{i}$. Thus for $i$ sufficiently large, $\mathcal{L}_{i}$ has self intersection which is a contradiction.

Let $\epsilon_{1}>0$, be such that $N_{P T(S)}\left(\mu, \epsilon_{1}\right) \subset W_{2}$. Again by Corollary 1.11 for $i$ sufficiently large, $\hat{\mathcal{E}}\left(\tau_{i}\right) \subset N_{P T(S)}\left(\mu, \epsilon_{1} / 2\right)$, where $\hat{\mathcal{E}}\left(\tau_{i}\right) \subset P T(S)$ is the union of geodesics corresponding to elements of $\mathcal{E}\left(\tau_{i}\right)$. Let $j$ be one such $i$.

We complete the proof of the theorem where the role of $V$ (as in the first paragraph) is played by $E_{j}$. Recall that $V\left(\tau_{j}\right)$ (resp. $\operatorname{int}\left(V\left(\tau_{j}\right)\right)$ is the set of measured laminations carried (resp. fully carried) by $\tau_{j} . \tau_{j}$ is transversely recurrent since it is an unzipping of $\tau_{1}$, it is recurrent since it carries $\mu$ and it is maximal since $\mu$ is maximal. Therefore in the terminology of [15, page 27], $\tau_{j}$ is complete and hence by [15, Lemma 3.1.2] $\operatorname{int}\left(V\left(\tau_{j}\right)\right)$ is open in $\mathcal{M L}(S)$. Let $\mu_{0}=\mu$ and $\mu_{1} \in E_{j}$ and for $i=0,1$ pick $\lambda_{i} \in \operatorname{int}\left(V\left(\tau_{j}\right)\right)$ so that $\phi\left(\lambda_{i}\right)=\mu_{i}$. Each $\lambda \in V\left(\tau_{j}\right)$ is determined by a transverse measure on the branches of $\tau_{j}$ and conversely. Also each of $\lambda_{0}, \lambda_{1}$ induces a positive transverse measure on each branch of $\tau_{j}$. Therefore we can define $e:[0,1] \rightarrow \operatorname{int}\left(V\left(\tau_{j}\right)\right)$ to be the straight line path from $\lambda_{0}$ to $\lambda_{1}$. Let $f:[0,1] \rightarrow \mathcal{M L}(S)$ be a PL almost filling path that closely approximates $e$ and connects its endpoints. Closely means that for each $t, f(t) \in \operatorname{int}\left(V\left(\tau_{j}\right)\right)$. Using $\epsilon=\epsilon_{1} / 2$ apply Lemma 5.1 to obtain the path $g:[0,1] \rightarrow \mathcal{E} \mathcal{L}(S)$. We show that $g([0,1]) \subset E_{1} \subset U$. With notation as in Lemma 5.1, if $s \in[0,1]$, then there exists $t \in[0,1]$ such that $\mathcal{L}^{*}(t) \subset N_{P T(S)}\left(g^{\prime}(s), \epsilon\right)$, for some diagonal extension $g^{\prime}(s)$ of $g(s)$. Since $\mathcal{L}^{*}(t)$ is carried by $\tau_{j}$, each point $x$ in $\mathcal{L}^{*}(t)$ is $\epsilon_{1} / 2$ close in $P T(S)$ to a point of $\mu$ and by Lemma 5.1 some point $y$ of $g^{\prime}(s)$ is $\epsilon$ close to $x$, we conclude $d_{P T(S)}(y, z) \leq \epsilon_{1}$ and hence $g^{\prime}(s) \cap W_{2} \neq \varnothing$. Therefore all sublaminations of $g^{\prime}(s)$ are fully carried by $\tau_{1}$, so $g(s)$ is fully carried by $\tau_{1}$. This completes the proof when $\mu$ is maximal.

The general case follows as above except that we must use the various diagonal extensions of $\mu$ and its associated train tracks. Here are more details. Let $U \subset \mathcal{E} \mathcal{L}(S)$ be open with $\mu \in U$ and let $\lambda \in \mathcal{M L}(S)$ such that $\phi(\lambda)=\mu$. Let $\tau_{1}, \tau_{2}, \ldots$ as above. By dropping a finite number of initial terms we can assume that each $\tau_{i}$ has exactly $n$ birecurrent diagonal extensions, $\tau_{i}^{1}, \ldots, \tau_{i}^{n}$ and for each $k$, the sequence 
$\tau_{1}^{k}, \tau_{2}^{k}, \ldots$ is a full unzipping sequence. Furthermore, $\bigcap \mathcal{E}\left(\tau_{i}^{k}\right)=\mathcal{E}\left(\mu^{k}\right)$ where $\mu^{k}$ is a diagonal extension of $\mu$. By an elementary topological argument, each such $\mu^{k}$ is carried by some $\left\{\tau_{i}^{q}\right\}$ sequence where each $\tau_{i}^{q}$ is maximal. (Compare with [5] which shows that a birecurrent train track is contained in a maximal such track.) Let $E_{i}=\left\{\mathcal{G} \in \mathcal{E} \mathcal{L}(S) \mid \mathcal{G}\right.$ is fully carried by a $\left.\tau_{i}^{k}\right\}$. Each $E_{i}$ is open in $\mathcal{E} \mathcal{L}(S)$ and by Corollary 1.11 after dropping the first $N$ terms of the various sequences we can assume that $E_{1} \subset U$.

There exists $W_{2}$ open in $P T(S)$ such that $\mu \subset W_{2}$ and if $\mathcal{L} \in \mathcal{L}(S)$ with $\mathcal{L} \cap W_{2} \neq \varnothing$, then every minimal sublamination of $\mathcal{L}$ is fully carried by one of $\tau_{1}^{1}, \ldots, \tau_{1}^{n}$. Since each diagonal of a $\mu^{k}$ is dense in $\mu$, there exists $W_{2}^{1}, \ldots, W_{2}^{n}$ open in $P T(S)$ such that for each $k, \mu^{k} \subset W_{2}^{k}$ and if $\mathcal{L} \cap W_{2}^{k} \neq \varnothing$, then every minimal sublamination of $\mathcal{L}$ is fully carried by one of $\tau_{1}^{1}, \ldots, \tau_{1}^{n}$. Let $\epsilon_{1}>0$, be such that $N_{P T(S)}\left(\mu^{k}, \epsilon_{1}\right) \subset W_{2}^{k}$ for each $k$. Let $j$ be so that for each $k, \widehat{\mathcal{E}}\left(\tau_{j}^{k}\right) \subset N_{P T(S)}\left(\mu^{k}, \epsilon_{1} / 2\right)$.

Let $\mu_{0}=\mu$ and $\mu_{1} \in E_{j}$. Let $\tau_{j}^{k}$ be a maximal track which carries $\mu_{1}$. For $i=0,1$ pick $\lambda_{i} \in V\left(\tau_{j}^{k}\right)$ so that $\phi\left(\lambda_{i}\right)=\mu_{i}$. Define $e:[0,1] \rightarrow V\left(\tau_{j}^{k}\right)$ to be the straight line path from $\lambda_{0}$ to $\lambda_{1}$. Since $V\left(\tau_{j}^{k}\right)$ is a PL-ball of codimension-0, and generic PL paths are almost filling, there exists $f:[0,1] \rightarrow V\left(\tau_{j}^{k}\right)$ a PL almost filling path from $\lambda_{0}$ to $\lambda_{1}$. Using $\epsilon=\epsilon_{1} / 2$ apply Lemma 5.1 to obtain the path $g:[0,1] \rightarrow \mathcal{E L}(S)$. We show that $g([0,1]) \subset E_{1} \subset U$. With notation as in Lemma 5.1, if $s \in[0,1]$, then $\mathcal{L}^{*}(t) \subset N_{P T(S)}\left(g^{\prime}(s), \epsilon\right)$, for some diagonal extension $g^{\prime}(s)$ of $g(s)$. Since $\mathcal{L}^{*}(t)$ is carried by $\tau_{j}^{k}$, each point $x$ in $\mathcal{L}^{*}(t)$ is $\epsilon_{1} / 2$ close in $P T(S)$ to a point of $\mu^{k}$ and by Lemma 5.1 some point $y$ of $g^{\prime}(s)$ is $\epsilon$ close to $x$, we conclude $d_{P T(S)}(y, z) \leq \epsilon_{1}$ and hence $g^{\prime}(s) \cap W_{2}^{k} \neq \varnothing$. Therefore each minimal sublamination of $g^{\prime}(s)$ is fully carried by a $\tau_{1}^{m}$, so $g(s)$ is fully carried by some $\tau_{1}^{k}$.

\section{$7 \mathcal{E} \mathcal{L}(S)$ is cyclic}

The main result of this section is the following:

Theorem 7.1 If $S$ is as in the hypothesis of Theorem 0.1 and $\mu \in \mathcal{E L}(S)$, then there exists a simple closed curve in $\mathcal{E} \mathcal{L}(S)$ passing through $\mu$.

Lemma 7.2 If $\mu \in \mathcal{E} \mathcal{L}(S)$, then there exists an embedding $f:[-1,1] \rightarrow \mathcal{E} \mathcal{L}(S)$ such that $f(0)=\mu$.

Proof Let $\lambda \in \mathcal{M L}(S)$ be such that $\phi(\lambda)=\mu$. Let $\sigma \subset \mathcal{M L}(S)$ be the measures supported on $\mu$. Let $\tau$ be a complete train track such that $\mu \subset \operatorname{int}\left(V_{\tau}\right)$. By choosing a generic straight line path $f:[-1,1] \rightarrow \operatorname{int}\left(V_{\tau}\right) \subset \mathcal{M L}(S)$ with $f(0)=\lambda$ we obtain a 
path in $\mathcal{A M L}(S)$ transverse to each $\widehat{B}_{\alpha}$ disjoint from each $\partial \widehat{B}_{\alpha}$, such that $f^{-1}(\sigma)=$ $\mu$ and $f(\partial[-1,1]) \in \mathcal{E} \mathcal{L}(S)$. Let $f^{*}(t)$ denote the almost minimal almost filling sublamination of $\phi(f(t))$.

We say that two geodesic laminations cross if they have a point of transverse intersection. We show that if $t>0$ and $s<0$, then $f^{*}(t)$ crosses $f^{*}(s)$. If not, being almost minimal almost filling, they must coincide. Since $f([0,1])$ is disjoint from the $\partial \widehat{B}_{\alpha}$ 's this implies that $\phi(f(t))=\phi(f(s))$. Since $\phi(f(t))$ is carried by $V(\tau)$ the straight line segment from $f(s)$ to $f(t)$ consists of measures on the same underlying lamination. Now either $f(t) \cup f(s) \subset \widehat{B}_{\alpha}$, some $\alpha$ or $f^{*}(s)=f^{*}(t) \in \mathcal{E L}(S)$. The former contradicts the fact that $f$ is transverse to $\widehat{B}_{\alpha}$, while the latter contradicts the condition $f^{-1}(\sigma)=\mu$.

We next show that there exists a path $g:[0,1] \rightarrow \mathcal{E L}(S)$ such that $g(0)=\mu$ and $g(1)=\phi(f(1))$ and for each $t>0$ and $s<0, g(t)$ crosses $f^{*}(s)$. Let $1=t_{0}>t_{1}>\cdots$ be a sequence such that $\operatorname{Lim} t_{i}=0$ and for all $i, \phi\left(f\left(t_{i}\right)\right) \in \mathcal{E} \mathcal{L}(S)$. It follows from Lemma 5.1 that for each $i \geq 1$, there exists a path $g_{i}:\left[t_{i}, t_{i-1}\right] \rightarrow \mathcal{E} \mathcal{L}(S)$ with $g_{i}\left(t_{j}\right)=\phi\left(f\left(t_{j}\right)\right), j \in\{i, i-1\}$ such that for $s<0$ and $t \in\left[t_{i}, t_{i-1}\right], g_{i}(t)$ crosses $f^{*}(s)$. Lemma 5.1 together with Proposition 3.2 and the minimality of $\mu$ implies that the $g_{i}$ 's can be further chosen so that given a neighborhood basis $\left\{U_{k}\right\}$ of $\mu$ in $\mathcal{E L}(S)$ and $n>0$, there exists $N(n)$ so that $i>N(n)$ implies that $g\left(\left[t_{i}, t_{i-1}\right]\right) \in U_{N}$. By concatenating these $g_{i}$ 's together and decreeing $g(0)=\mu$ we obtain the desired function $g$.

By Lemma 7.3 stated below, there exists an embedded path $h:[0,1] \rightarrow g([0,1]) \subset \mathcal{E} \mathcal{L}(S)$ from $\mu$ to $g(1)$. Since each $h(t)$ crosses $\phi\left(f^{*}(s)\right)$ for $s<0$, we can repeat this argument to produce an embedded path $h:[-1,0] \rightarrow \mathcal{E L}(S)$ from $\phi(f(-1))$ to $\mu$ such that if $s<0$ and $t>0$, then $h(s)$ crosses $h(t)$. It follows that $h:[-1,1] \rightarrow \mathcal{E L}(S)$ is an embedded path through $\mu$.

Lemma 7.3 If $X$ is Hausdorff and $g:[0,1] \rightarrow X$ is continuous and $p, q \in g([0,1])$, then there exists an embedded path in $g[0,1]$ from $p$ to $q$.

Proof This is [21, Corollary III.3.11] which asserts that any two points of a Peano continuum $C$ are connected by an embedded path in $\mathrm{C}$, where a Peano continuum is the image of a continuous map of $[0,1]$ into a Hausdorff space. See [21, III.1 and III.1.29] for this characterization of Peano continuum.

Proof of Theorem 7.1 Apply Lemma 7.2 to find an embedded path $h:[-1,1] \rightarrow$ $\mathcal{E L}(S)$ with $h(0)=\mu$. Let $\lambda_{t} \in \mathcal{M L}(S)$ be such that $\phi\left(\lambda_{t}\right)=h(t)$ for $t \in\{-1,1\}$. Again let $\sigma \subset \mathcal{M L}(S)$ denote the measures on $\mu$. Since $\sigma$ is a cone on a closed 
simplex, it follows that there exists a path $f:[-1,1]$ in $\mathcal{M L}(S)$ from $\lambda_{-1}$ to $\lambda_{1}$ disjoint from $\sigma$. After a small homotopy we can assume that $f$ is a PL almost filling path. For each $t \in[-1,1], f^{*}(t)$ crosses $\mu$ so by Proposition 3.2 there exists $\epsilon>0$ so that for $|s|<\epsilon, t \in[-1,1], f^{*}(t)$ crosses $h(s)$. The proof of Lemma 7.2 produces an embedded path $k:[-1,1] \rightarrow \mathcal{E} \mathcal{L}(S)$ from $h(-1)$ to $h(1)$ with this same property. Concatenating this path with $h \mid[-1,-\epsilon]$ and $h \mid[\epsilon, 1]$ we obtain a path $\kappa:[a, b] \rightarrow \mathcal{E} \mathcal{L}(S)$ from $h(-\epsilon)$ to $h(\epsilon)$ which intersects $h \mid[-\epsilon, \epsilon]$ only at its endpoints. Apply Lemma 7.3 to obtain an embedded path with the same properties. Fusing this path with $h \mid[-\epsilon, \epsilon]$ we obtain the desired embedding of $S^{1}$ into $\mathcal{E} \mathcal{L}(S)$ passing through $\mu$.

Corollary 7.4 $\mathcal{E} \mathcal{L}(S)$ has no cut points.

\section{Questions}

Question 8.1 If $\lambda$ and $\lambda^{\prime} \in \mathcal{P} \mathcal{M L}(S)$ satisfy $\phi(\lambda), \phi\left(\lambda^{\prime}\right) \in \mathcal{E} \mathcal{L}(S)$, is there a filling path in $\mathcal{P} \mathcal{M L}(S)$ connecting them?

Question 8.2 Is $\mathcal{E} \mathcal{L}(S) n$-connected if $\mathrm{S}$ has sufficiently high complexity?

\section{Applications}

An unsuccessful attempt to find a positive solution to Question 8.1 led to the interesting study of how various $B_{\beta}$ 's can intersect a given $B_{\alpha}$ which in turn led to a new construction of nonuniquely ergodic measured laminations in any finite type surface $S$ not the 1 -holed torus or 3 - or 4-holed sphere.

Theorem 9.1 If $\alpha_{1}, \ldots, \alpha_{n}$ are pairwise disjoint simple closed geodesics in the complete, hyperbolic, finite type, orientable surface $S$, and for each $i, U_{i}$ is an open set in $\mathcal{P M L}(S)$ about $\alpha_{i}$ then there exists a $(n-1)-\operatorname{simplex} \Sigma$ in $\mathcal{P} \mathcal{M L}(S)$ representing a filling nonuniquely ergodic measured lamination with the endpoints of $\Sigma$ respectively in $U_{1}, \ldots, U_{n}$.

Proof In the statement of the theorem as well as in the proof, simple closed curves are naturally identified with points in $\mathcal{P} \mathcal{M L}(S)$.

We provide the argument for the case $n=2$. The proof in the general case is similar. Let $\alpha$ and $\beta$ (resp. $U_{\alpha}$ and $U_{\beta}$ ) denote $\alpha_{1}$ and $\alpha_{2}$ (resp. $U_{1}$ and $U_{2}$ ). Let $a_{1}$ be a simple closed geodesic disjoint from $\beta$ and close to $\alpha$. For example, let $a_{1}^{\prime} \neq \alpha$ be any geodesic disjoint from $\beta$ which intersects $\alpha$ and let $a_{1}$ be obtained from $a_{1}^{\prime}$ by 
doing a high power Dehn twist to $\alpha$. Let $b_{1}$ a simple closed geodesic disjoint from $a_{1}$ and very close to $\beta$. Let $a_{2}$ a simple closed geodesic disjoint from $b_{1}$ and extremely close to $a_{1}$ and let $b_{2}$ a simple closed geodesic disjoint from $a_{2}$ and super close to $b_{1}$. Continuing in this manner we obtain the sequence $a_{1}, a_{2}, a_{3}, \ldots$ which converges to $\lambda_{\alpha}$ and the sequence $b_{1}, b_{2}, \ldots$ converges to $\lambda_{\beta}$ where $\lambda_{\alpha}, \lambda_{\beta}$ respectively lie in $U_{\alpha}$ and $U_{\beta}$.

Since intersection number is continuous on $\mathcal{M L}(S)$, and for each $i, a_{i}$ and $b_{i}$ are pairwise disjoint it follows that $i\left(\lambda_{\alpha}, \lambda_{\beta}\right)=0$. We now show that with a little care in the choice of the $a_{i}$ 's and $b_{i}$ 's that $\phi\left(\lambda_{\alpha}\right)=\phi\left(\lambda_{\beta}\right) \in \mathcal{E L}(S)$. Let $V_{\alpha} \subset U_{\alpha}$ and $V_{\beta} \subset U_{\beta}$ be closed neighborhoods of $\alpha$ and $\beta$. We argue as in the proof of Lemma 4.3. Recall that $C_{1}, C_{2}, \ldots$ is an enumeration of the simple closed geodesics in $S$. If possible choose $a_{1}$ to intersect $C_{1}$ transversely. We can always do that and have $a_{1}$ as close to $\alpha$ as we like, unless $C_{1}=\beta$ or $\beta$ separates $C_{1}$ from $\alpha$. If we must have $a_{1} \cap C_{1}=\varnothing$, then we can choose $b_{1} \in \operatorname{int}\left(V_{\beta}\right)$ so that $b_{1} \cap C_{1} \neq \varnothing$ and hence in any case, $a_{2}$ can be chosen so that $a_{2} \cap C_{1} \neq \varnothing$ and $a_{2}$ is close to $\alpha$. Let $V_{2} \subset V_{\alpha}$ a closed neighborhood of $a_{2}$ in $\mathcal{P M L}(S)$ so that if $x \in V_{2}$, then $x$ and $C_{1}$ have nontrivial intersection number. As above, choose $b_{2}, a_{3}, b_{3}, a_{4}$ so that $a_{4} \cap C_{2} \neq \varnothing, a_{3} \cup a_{4} \subset \operatorname{int}\left(V_{2}\right)$ and $b_{2} \cup b_{3} \subset \operatorname{int}\left(V_{\beta}\right)$. Choose $V_{4} \subset V_{2}$ a closed neighborhood of $a_{4}$ so that $x \in V_{4}$ implies that $x$ and $C_{2}$ have nontrivial intersection number. Inductively construct $b_{4}, b_{5}, \ldots, a_{5}, a_{6}, \ldots$ If after passing to subsequence $\lambda_{\alpha}=\operatorname{Lim} a_{i}$, then for all $j, i\left(\lambda_{\alpha}, C_{j}\right) \neq 0$ and hence $\phi\left(\lambda_{\alpha}\right) \in \mathcal{E} \mathcal{L}(S)$. By construction $\lambda_{\alpha} \in U_{\alpha}$. Also, if after passing to subsequence $\lambda_{\beta}=\operatorname{Lim} b_{i}$, then $\lambda_{\beta} \in U_{\beta}$. Since $\lambda_{\alpha} \in \mathcal{E} \mathcal{L}(S)$ and has trivial intersection number with $\lambda_{\beta}$, it follows that $\phi\left(\lambda_{\alpha}\right)=\phi\left(\lambda_{\beta}\right)$. The projection to $\mathcal{P} \mathcal{M L}(S)$ of a straight line segment in $\mathcal{M L}(S)$ connecting representatives of $\lambda_{\alpha}$ and $\lambda_{\beta}$ gives the desired 1-simplex $\Sigma$.

Kasra Rafi and Saul Schleimer [16] use Theorem 0.1 to prove the following two interesting rigidity results.

Theorem 9.2 (Rafi-Schleimer) Let $S$ be a finite type orientable surface of negative Euler characteristic which is not the 3-holed sphere, 4-holed sphere or 1-holed torus. Every quasi-isometry of $\mathcal{C}(S)$ is bounded distance from a simplicial automorphism of $\mathcal{C}(S)$. Consequently, QI $(\mathcal{C}(S)$ ) the group of quasi-isometries (modulo bounded quasiisometries) of $\mathcal{C}(S)$ is isomorphic to $\operatorname{Aut}(\mathcal{C}(S))$ the group of simplicial automorphisms.

Theorem 9.3 (Rafi-Schleimer) Let $S$ be a finite type orientable surface of negative Euler characteristic which is not the 3-holed sphere, 4-holed sphere or 1-holed torus. Suppose that neither $S$ nor $\Sigma$ is the surface of genus-2 or the twice-punctured surface of genus-1, then $S$ and $\Sigma$ are homeomorphic if and only if $\mathcal{C}(S)$ is quasi-isometric to $\mathcal{C}(\Sigma)$. 
Chris Leininger and Saul Schleimer [12] give a detailed proof of the following Theorem 9.4 which they say "seems to be well known", that is a consequence of many deep results in Kleinian group theory. Using their notation, the space of doubly degenerate Kleinian surface groups is denoted by $\mathrm{DD}(S, \partial S)$ and equals $\{M \in \mathrm{AH}(S, \partial S) \mid \mathcal{E}(M) \in$ $\mathcal{E} \mathcal{L}(S) \times \mathcal{E} \mathcal{L}(S)\}$. Here $\mathcal{E}(M)$ is the end invariant of the doubly degenerate group $M$ and is an element of $\mathcal{E} \mathcal{L}(S) \times \mathcal{E} \mathcal{L}(S)-\Delta$, where $\Delta$ is the diagonal.

Theorem $9.4[12$, Theorem 6.5] $\mathcal{E}: \operatorname{DD}(S, \partial S) \rightarrow \mathcal{E L}(S) \times \mathcal{E} \mathcal{L}(S) \backslash \Delta$ is a homeomorphism.

Theorem 9.5 If $S$ is a finite type hyperbolic surface, not the once-punctured torus or 3-or 4-punctured sphere, then $\mathcal{E} \mathcal{L}(S) \times \mathcal{E} \mathcal{L}(S) \backslash \Delta$ is path connected, locally path connected and cyclic.

Proof Local path connectivity is immediate. Let $\sigma_{0}, \sigma_{1}, \mu$ be distinct elements of $\mathcal{E L}(S)$. The method of proof of Theorem 7.1 shows how to construct a path from $\sigma_{0}$ to $\sigma_{1}$ disjoint from $\mu$ and an embedded circle through $\sigma_{0}$ disjoint from $\mu$. Using this it is routine to show path connectivity and cyclicity.

Corollary 9.6 $\operatorname{DD}(S, \partial S)$ is path connected, locally path connected and cyclic. if $S$ is a compact orientable hyperbolic surface that is not the sphere with 3 or 4 open discs removed or the torus with an open disc removed.

\section{References}

[1] B H Bowditch, Intersection numbers and the hyperbolicity of the curve complex, J. Reine Angew. Math. 598 (2006) 105-129 MR2270568

[2] R D Canary, D B A Epstein, P Green, Notes on notes of Thurston, from: "Analytical and geometric aspects of hyperbolic space (Coventry/Durham, 1984)", (D B A Epstein, editor), London Math. Soc. Lecture Note Ser. 111, Cambridge Univ. Press (1987) 3-92 MR903850

[3] A Fathi, F Laudenbach, V Poenaru (editors), Travaux de Thurston sur les surfaces, second edition, Astérisque 66, Société Mathématique de France, Paris (1991) MR568308 Séminaire Orsay, With an English summary

[4] W Goldman, W P Thurston, Lecture notes from Boulder (1981)

[5] U Hamenstädt, Train tracks and mapping class groups I, to appear in Invent. Math.

[6] U Hamenstädt, Train tracks and the Gromov boundary of the complex of curves, from: "Spaces of Kleinian groups", (Y N Minsky, M Sakuma, C Series, editors), London Math. Soc. Lecture Note Ser. 329, Cambridge Univ. Press (2006) 187-207 MR2258749 
[7] W J Harvey, Boundary structure of the modular group, from: "Riemann surfaces and related topics: Proceedings of the 1978 Stony Brook Conference (1978)", (I Kra, B Maskit, editors), Ann. of Math. Stud. 97, Princeton Univ. Press (1981) 245-251 MR624817

[8] A Hatcher, W P Thurston, A presentation for the mapping class group of a closed orientable surface, Topology 19 (1980) 221-237 MR579573

[9] R Kent IV, C Leininger, Shadows of mapping class groups: capturing convex cocompactness, to appear in Geom. Funct. Anal.

[10] E Klarreich, The boundary at infinity of the curve complex and the relative mapping class group, Preprint

[11] C Leininger, $\mathbf{M} \mathbf{M j}$, S Schleimer, Cannon-Thurston maps and the curve complex, Preprint

[12] C Leininger, S Schleimer, Connectivity of the space of ending laminations, Preprint

[13] H A Masur, Y N Minsky, Geometry of the complex of curves. I. Hyperbolicity, Invent. Math. 138 (1999) 103-149 MR1714338

[14] L Mosher, Train track expansions of measured foliations, Preprint

[15] R C Penner, J L Harer, Combinatorics of train tracks, Annals of Math. Studies 125, Princeton Univ. Press (1992) MR1144770

[16] K Rafi, S Schleimer, Curve complexes with connected boundary are rigid, Preprint

[17] S Schleimer, The end of the curve complex, Preprint

[18] W P Thurston, The geometry and topology of three-manifolds, Princeton Univ. Math. Dept. Lecture Notes (1980) Available at http://msri.org/publications/books/ gt3m/

[19] W P Thurston, Minimal stretch maps between hyperbolic surfaces, Unpublished preprint (1986) arXiv:math.GT/9801039

[20] W P Thurston, On the geometry and dynamics of diffeomorphisms of surfaces, Bull. Amer. Math. Soc. (N.S.) 19 (1988) 417-431 MR956596

[21] R L Wilder, Topology of manifolds, Amer. Math. Soc. Colloq. Publ. 32, Amer. Math. Soc. (1979) MR598636 Reprint of 1963 edition

[22] X Zhu, F Bonahon, The metric space of geodesic laminations on a surface. I, Geom. Topol. 8 (2004) 539-564 MR2057773

Department of Mathematics, Princeton University

Princeton, NJ 08544, USA

gabai@princeton.edu

Proposed: Benson Farb

Seconded: Jean-Pierre Otal, Tobias Colding
Received: 5 September 2008 Revised: 3 January 2009 